\title{
The effect of bureaucratic corruption on household food insecurity: evidence from Sub-Saharan Africa
}

\author{
Olayemi M. Olabiyi ${ }^{1}$
}

Received: 20 January 2021 / Accepted: 9 October 2021 / Published online: 28 October 2021

(c) International Society for Plant Pathology and Springer Nature B.V. 2021

\begin{abstract}
Corruption is a worldwide phenomenon, but the crisis seems to have no bounds in Sub-Saharan Africa, where the absurdity has penetrated and continues to impair the public service sector. As long as the anomaly continues, two outcomes are inevitable. First, an average member of the public runs the risk of paying more for any government service. Second, some or all resources earmarked for other essential needs might get sacrificed to pay for a needed public service. Such unforeseen adverse shocks could potentially increase the chances of wicked problems such as food insecurity. Consequently, this paper examined the welfare effect of bureaucratic corruption on households' ability to procure food in a safe and socially acceptable manner in sub-Saharan Africa using pooled Afrobarometer micro-level data from Rounds 5, 6, and 7 spanning 2011 to 2018. Estimates from logistic regression and the endogenous dummy variable regression that addressed the endogeneity of bureaucratic corruption in the food insecurity model revealed that bureaucratic corruption induced the experience of household food insecurity. Further analyses showed that some public entities have a more significant debilitating effect than others on household food security conditions. More importantly, any contact with government institutions causes household food security status to spiral downward. These results suggest that public institutions in Sub-Saharan Africa are weak and undoubtedly contribute to household food insecurity. Therefore, there is a need for a paradigm shift in how these entities serve the public or else a lasting solution to food insecurity might remain elusive in the region.
\end{abstract}

Keywords Bureaucratic corruption · Household food insecurity · Sub-Saharan Africa · Afrobarometer Survey

\section{Introduction}

Corruption is a symptom of a deeper and more general malaise in any society. While it is not peculiar to any country or region, it tends to be more prevalent in Sub-Saharan Africa. The majority of the countries in this region regularly appear in the most corrupt countries cadre of the Corruption Perceptions Index. According to the Transparency International, 2019 report, only seven countries in the region scored above the global average in the Corruption Perceptions Index for the year. Undoubtedly, the issue has several dimensions, including the rent-seeking behaviour of public officials, also known as bureaucratic corruption (Ksenia, 2008).

Olayemi M. Olabiyi

olayemi.olabiyi@sait.ca

1 School of Business, Southern Alberta Institute of Technology, 1301 - 16 Ave NW, Calgary, AB T2M 0L4, Canada
Like in other regions, it is unlawful to request or accept a bribe in countries across Sub-Saharan Africa. Notwithstanding, the practice is widespread (Médard, 2017), but only a handful of the infractions tends to be reported for controls or sanctions. For example, a quick rundown of Round 6 of the Afrobarometer survey revealed that more than 90 percent of bureaucratic corruption cases were unreported to the appropriate quarters. Moreover, for reported cases, only 27.4 percent of all respondents affirmed that the relevant authorities took decisive actions on corrupt public officials (Afrobarometer, 2016).

As long as sanctions remain weak or non-existent, corrupt public employees might have no reason to stop surcharging the public, especially the victims of misfortunes (Guriev, 2004; Hunt, 2007; Justesen \& Bjornskov, 2014; Peiffer \& Rose, 2018; Shleifer \& Vishny, 1993). However, these demands might compel the vulnerable population needing government services to sacrifice some or all resources earmarked for other basic needs (Hunt \& Laszlo, 2005). Such unexpected negative shock to household finances 
could likely, among other things, constrict the low-income households' food security status, or worse still, their ability to procure food through a socially acceptable approach. While other developing regions have made significant strides in decreasing the food-insecure population, the reverse is the case across Sub-Saharan African countries (FAO et al., 2019; George et al., 2020; de Beer et al., 2020). The reason is that more than fifty percent of the Sub-Saharan African population constantly experiences moderate to severe difficulty procuring food in an ethically agreeable manner (FAO et al., 2020).

Given that most of the Sub-Saharan African population lives on less than $\$ 2$ a day and are more easily intimidated into paying a bribe (Hunt \& Laszlo, 2012), there is little reason to expect that bureaucratic corruption has no consequences on food security status. Consequently, this paper aims to fill the corresponding gap in the literature by investigating three issues pertaining to the relationship between bureaucratic corruption and household food insecurity in Sub-Saharan Africa using micro-level data. First, it examines whether households that paid bribes are susceptible to being food insecure. Second, it explores whether any or all bureaucratic corruption sources are benign to household food security status. Third, it investigates whether variations in the bureaucratic corruption burden have differing implications for households that reported being always, moderately, or slightly food insecure. The rest of this paper is structured as follows: Sect. 2 encapsulates the literature review on the drivers of food insecurity and the consequences of bureaucratic corruption. Section 3 describes the data and method, while Sect. 4 presents the results and discusses the findings. Finally, Sect. 5 concludes the study.

\section{Literature review}

Numerous studies have investigated the food insecurity problem. Their findings revealed, among other things, that the phenomenon could be chronic or transitory and that both are sensitive to livelihood stressors (Verpoorten et al., 2013). One such stressor is abrupt adverse shocks such as job loss, climate change, armed conflict, insurgencies, price hikes, and the COVID-19 lockdown (Leete and Bania 2010; D'Souza and Jolliffe 2013; George et al., 2020; Ngcamu and Chari 2020; Arndt et al., 2020). Regarding income shock, Leete and Bania (2010) showed that any unexpected decline in monthly income drastically reduced food security status across all households but resulted in more debilitating consequences for household structures with less economic resilience. Specifically, they found that the expected likelihood of food insecurity occurring in single-mother families ranged from 3.6 percent to 4.9 percent. Likewise, the possibility of the phenomenon among households with incomes below 200 percent of the poverty level ranged from 4.4 percent to 5.3 percent. Similarly, Dahl et al. (2014) revealed that families that experienced significant income drops had a 31 percent chance of experiencing food insecurity in the United States.

Mabiso et al. (2014) asserted that volatility in food prices could engender erratic caloric intake of healthy foods, especially in rural Mozambique households whose primary source of income comes from non-farm employment. Using self-reported data of more than 50,000 individuals in eighteen Sub-Saharan African countries, Verpootenet al (2013) investigated how the global food price shock of 2005-2008 moderated household food security status. They found that the adverse event positively affected rural families while it negatively affected urban households. Specifically, the authors showed that the depth of food insecurity during the global food crisis decreased by 9.2 percent in rural areas but increased substantially, by 7.8 percent, in urban areas from 2005 to 2008. In a related vein, Matz et al (2015) found that not all food price hikes worsen household security status in Ethiopia. They showed that the volatility in the price of teff caused the poor in Ethiopia to lower their number of daily meals and switch to less-preferred options. Further analysis revealed that the urban poor were highly susceptible to skipping or missing meals than their rural counterparts.

Unlike volatility of food prices, which had a reducing effect on urban household food security status, the reverse is the case for climate change, which tends to be more concerning in rural areas. Using mixed methods, Asare-Nuamah (2021) asserted that climate variability constricted agricultural production in rural Ghana and caused households living in those areas, many of whom were into subsistence agriculture, to lack the quantity and quality of healthy food needed for consumption. However, even in rural areas, the extent of the effect tends to depend on the proportion of the potential loss suffered to other adverse shocks. For example, Del Ninno and Marini (2005) found that rural Zambian households that have the likelihood of losing more than ten percent of their income would struggle to survive in the face of a drought.

Like other adverse shocks, bribery interferes with resource allocation, economic stability, and income distribution, which widens the income inequality gap and fans poverty among most citizens (Cooray \& Schneider, 2016; Gupta et al., 2002). Consequently, individuals living in corruption-ridden countries often feel less satisfied with their lives than their counterparts in relatively corruption-free countries (Helliwell, 2003; Tavits, 2008). Using micro-level data, Sulemana et al. (2017) showed that bribery is a double-edged sword that negatively affects both the giver and the receiver. They found that the wellbeing of givers decreased by $0.05-0.08$ units while that of receivers decreased by 0.08 , which are coincidentally the same magnitude. Mbate (2018) lends support to 
the notion that the poor are highly vulnerable to paying bribes. The author showed that the poor in Kenya not only spent more of their resources on bribes than the non-poor, but the former paid them more frequently than the latter. If the poor pay bribes more regularly, wouldn't some resources earmarked for household expenditure such as food get sacrificed, thereby lowering diet quality? The following section explains the material and method employed to investigate the matter.

\section{Material and method}

The analyses conducted in this paper centred on pooled micro-level data from Rounds 5, 6, and 7 of the Afrobarometer Surveys, which spanned eight years, i.e., $2011-2018$. Rounds 5 occurred in 2011-2013, while Rounds 6 and 7 were conducted in 2014-2015 and 2016 - 2018, respectively. All the data were collected through face-to-face interviews. In each sample household, one voting-age respondent is asked questions about political, socioeconomic, democratic, and other related issues; and at all stages of data collection, random selection and probability proportionate to population size methods were used to obtain a representative sample size (Afrobarometer, 2011, 2016, 2018). Each round randomly interviewed 1200 people; however, some countries had 2400 sample sizes.

Given that Sub-Saharan Africa was the study's scope, Algeria, Egypt, Sudan, Tunisia, and Morocco were excluded because they were not part of the World Bank's classification of countries in that region. The number of countries surveyed oscillated between twenty-nine and thirty-one. Round 5 captured data from twenty-nine, ${ }^{1}$ whereas Rounds 6 and 7 each garnered data from thirty-one countries in the region. ${ }^{23}$ After excluding the missing observations in all variables of interest, the remaining sample size was 118,089 respondents.

\footnotetext{
${ }^{1}$ Round 5: Benin, Botswana, Burkina Faso, Burundi, Cameroon, Cabo Verde, Cote d'Ivoire, Ghana, Guinea, Kenya, Lesotho, Liberia, Madagascar, Malawi, Mali, Mauritius, Mozambique, Namibia, Niger, Nigeria, Senegal, Sierra Leone, South Africa, eSwatini, Tanzania, Togo, Uganda, Zambia, and Zimbabwe.

2 Round 6: Benin, Botswana, Burundi, Cabo Verde, Cameroon, Cote d'Ivoire, Ghana, Gabon, Mauritius, Malawi, Namibia, Togo, Lesotho, Tanzania, Kenya, Nigeria, Zambia, Zimbabwe, Madagascar, Mali, Senegal, eSwatini, Burkina Faso, Guinea, Liberia, Niger, Sierra Leone, Uganda, South Africa, Mozambique, Sao Tome and Principe.

${ }^{3}$ Round 7: Benin, Botswana, Burkina Faso, Cabo Verde, eSwatini, Gabon, Gambia, Ghana, Kenya, Lesotho, Liberia, Madagascar, Malawi, Mali, Mauritius, Mozambique, Namibia, Niger, Nigeria, Sao Tome and Principle, Senegal, Sierra Leone, South Africa, Tanzania, Togo, Uganda, Zimba and Zimbabwe.
}

\subsection{Dependent variable}

For this study, the outcome variable is the self-reported experience of food insecurity, which has five different outcomes. These were rearranged into first, a categorical variable, and afterward, an ordinal variable. For the binary setup, the variable assumed a value of one if a respondent reported having any food insecurity experience but zero otherwise. However, the ordinal outcomes were never, just once or twice, several times, many times, and always.

\subsection{The primary independent variable of interest}

The primary independent variable of interest, i.e., household exposure to bribery, is a by-product of five different questions in Rounds 5, 6, and 7. These questions probed how often, if ever, did the respondents in the past one year have to pay a bribe, give a gift, or do a favour for a government official in order to receive a document/permit, water sanitation/household services, medical care in a local health clinic or hospital, public school placement, police assistance, assistance from the court, or avoid problems with the police. The wording of these questions exempts the respondents from self-incrimination and places the blame on the public officials. In other words, the survey questions connote the bribe-payers as "victims of corruption" (Seglison, 2006). Each of these questions has five responses, i.e., never, once or twice, a few times, often, and no contact in the past year, and through them, binary and ordered measures emanated.

Regarding the binary measure, the primary independent variable of interest took the value of one if a respondent reported having any exposure to bribery but zero otherwise. When it comes to the ordered measures, the variable assumed five different categories. These are once or twice, a few times, often, no contact in the past year, and never.

\subsection{Control variables}

Also, the study controlled for other variables identified in the literature as determinants of household food insecurity. These are age, gender, education, asset, social capital, employment status, household size, location, time and location, i.e., country and regional effects.

\subsection{Methodology}

The empirical analyses of the pooled micro-level data employed three econometric approaches. The first technique was binary logistic regression. The probability of being food insecure conditional on the social and economic factors are represented as follows: 
$Y_{i j t}=\alpha_{1 j t} Z_{i j t}+\alpha_{2 j} X_{i j t}+\gamma_{t}++\varepsilon_{i j}$

$Y=\left\{\begin{array}{l}1, \text { if } Y_{i j t}>0 \\ 0, \text { otherwise }\end{array}\right.$

The logistic transformation of Eqs. 1 and 2 are presented as follows:

$\log \left(\frac{\pi_{i j t}}{1-\pi_{i j t}}\right)=\beta_{0}+\beta_{1 j} Z_{i j}+\beta_{2 j} X_{i j}+\gamma_{t}+\varepsilon_{i j}$

where $\pi_{i j t}=\mathrm{P}\left(\right.$ foodinsecure $\left._{i j t}=1\right), \quad 1-\pi_{i j t}=$ $\mathrm{P}\left(\right.$ foodinsecure $\left._{i j t}=0\right), Z_{i j}$ is a vector of individual-level controls (age, education, gender, employment status, geographical location, household size, asset, and community involvement), and $X_{i j t}$ is bureaucratic corruption (a binary variable, which equals one if an individual $i$ in country $j$ and year $t$ paid a bribe to access a public service and 0 otherwise).

Bureaucratic corruption could be an endogenous variable, which would render the logistic technique an inefficient estimator. For instance, bureaucratic corruption and education are correlated (Gupta et al., 2000). Consequently, the issue was addressed using the endogenous dummy-variable regression specified as follows:

$Y_{i j t}=x_{i j} \beta+\delta t_{i j}+\gamma_{t}+\lambda_{j}+\varepsilon_{i j}$

$t_{i j t}$ is a binary-instrumental variable and is assumed to stem from an unobservable latent variable:

$t_{i j t}^{*}=w_{i j} \gamma+u_{i j}$

$t_{i j t}=\left\{\begin{array}{l}1, \text { if } t_{i j t}^{*}>0 \\ 0, \text { otherwise }\end{array}\right.$

where:

$x_{i j t}$ are the covariates used to model the outcome, $w_{i j t}$ are the covariates used to model treatment assignment, $i$ is the index for individual effect, $j$ is the index for regional/country effect, $t$ is the survey year, $\gamma_{t}$ is the year effect, $\lambda_{j}$ is the location effect, i.e., country or regional, and the error terms $\varepsilon_{i j t}$ and $\mu_{i j t}$ are bivariate normal with mean zero and covariance matrix

$\left[\begin{array}{cc}\sigma^{2} & \rho \sigma \\ \rho \sigma & 1\end{array}\right]$

The analysis employed roadblocks by the police in the enumeration area as the instrumental variable because the existence of such increases the possibility of the public becoming victims of corruption (Foltz \& Opoku-Agyemang, 2015), but it does not directly impact household food security status. Appendix 1 revealed that the regional distribution of police roadblocks varied between food-secure and food-insecure precincts across Sub-Saharan Africa. However, police roadblocks were more prevalent in Central Africa. For instance, 15 percent of the food-insecure Central African respondents reported residing in neighbourhoods with police roadblocks. On the other hand, the proportion (14.4 percent) was slightly lower for those residing in food-secure locations across Central Africa. Consequently, the instrument is correlated with bureaucratic corruption but uncorrelated with the error term in the food insecurity model. Therefore, the covariates $\mathrm{x}_{\mathrm{ijt}}$ and $\mathrm{w}_{\mathrm{ijt}}$ are exogenous (Cameron \& Trivedi, 2005; Wooldridge, 2010).

Furthermore, multivariate ordered logistic regression analysis was employed to take advantage of the ranked nature of the outcome variable. The equation for multivariate ordered logistics is:

$\log \left(\frac{P\left(Y_{i j t} \leq 1\right.}{\left(1-P\left(Y_{i j t} \leq 1\right)\right.}\right)=\beta_{0}+\sum_{k=1}^{q-1} \beta_{j k} X_{i j k}+\varepsilon_{i j}$

where $P\left(Y_{i j t} \leq 1\right)$ is the probability that a respondent $i$ in country $j$ and year $t$ will report one form of food insecurity, $\left[\left(1-\mathrm{P}\left(Y_{i j t} \leq 1\right)\right]\right.$ is the probability that the respondent is food secure, $X_{i j k}$ are the independent variables, and $\varepsilon_{i j}$ is the error term (Atuoye et al., 2019).

\section{Findings and discussion}

Figure 1 illustrates the distribution of food insecurity and victims of bribery by regions. The proportion of respondents who reported being food insecure consists of those that experience the problem just once or twice, several times, many times, and always. On the other hand, the proportion of respondents who had unwillingly offered bribes or gifts to public officials captured three different categories: once or twice, a few times, and often. The regional classification was adopted to mitigate the variation in the number of countries in the pooled surveys. Of the four regions, West Africa had the fewest respondents that reported food insecurity. In contrast, about six out of every ten respondents in Central Africa reported being food insecure, making it the hotspot. Regarding forced bribery, Southern Africa recorded the lowest incidence of 15.9 percent, while East Africa had the highest incidence of 34.76 percent.

Figure 2 shows wide variation in the reported bribery and food insecurity by countries in Sub-Saharan Africa. In Cabo Verde, 6.9 percent of respondents reported paying at least one bribe in the past 12 months. On the other hand, 36 percent of respondents in Mozambique reported paying at least one bribe in the intervening period. Regarding food insecurity, three out of every ten respondents in $\mathrm{Cabo}$ Verde reported that they experienced the problem in diverse degrees. However, more than 60 percent of respondents in 
Fig. 1 Proportion of respondents who were victims of bribery and reported food insecurity by regions in sub-Saharan Africa

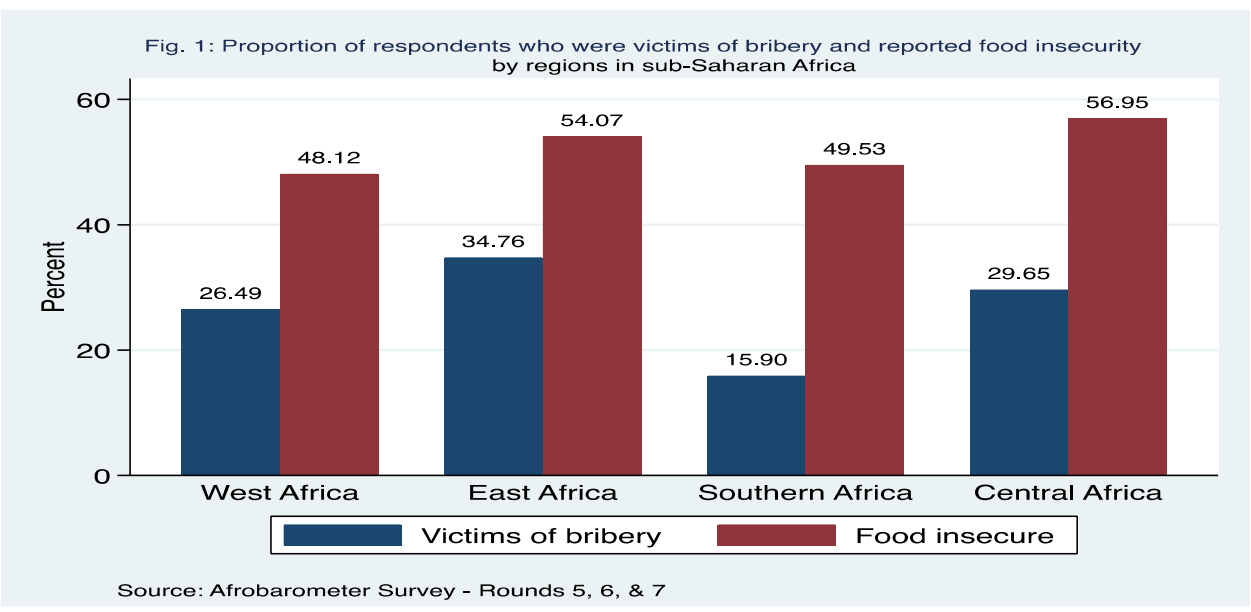

three countries, i.e., Nigeria, Senegal, and Sierra Leone, reported being food insecure. Except for Uganda, Mauritius, Togo, Guinea, and Ethiopia, more than fifty percent of respondents in each of the remaining selected countries, i.e., Mozambique, Namibia, South Africa, Tanzania, Zimbabwe, Togo, Burundi, Cameroon, and eSwatini, reported form of food insecurity. It is worth noting that sixty-four out of every 100 respondents in Senegal reported experiencing the problem just once or twice, several times, many times, or always, making it the hotspot.

The variable descriptions, along with their summary statistics, are shown in Table 1. The mean score for food insecurity was 2.060, which lies between 2 (= several times) and 3 (=many times), thereby suggesting that fifty percent of the respondents reported one form of food insecurity. Twenty-four percent of the respondents paid bribes before accessing publicly funded services, translating to about one out of every four respondents. Additionally, 16 percent, 9 percent, and 6 percent of the respondents reported paying bribes before accessing government services just once or twice, a few times, and often, respectively. Other respondents' attributes included but were not limited to the following: forty-one percent resided in urban areas, fifty percent were female, the average household comprised four people, the average educational attainment was completed primary education, and eighty-two percent owned at least one asset.

Table 2 details the results of the endogenous dummy variable and logistic regressions. Models I, II, III, and IV addressed the endogeneity of bribery. However, Models I and II have both year and regional effects, but the latter contained an interaction effect. Therefore, we retained the year effect in Models II and IV but replaced the region effect
Fig. 2 Fig. 1 Proportion of respondents who were victims of bribery and reported food insecurity by selected countries in sub-Saharan Africa

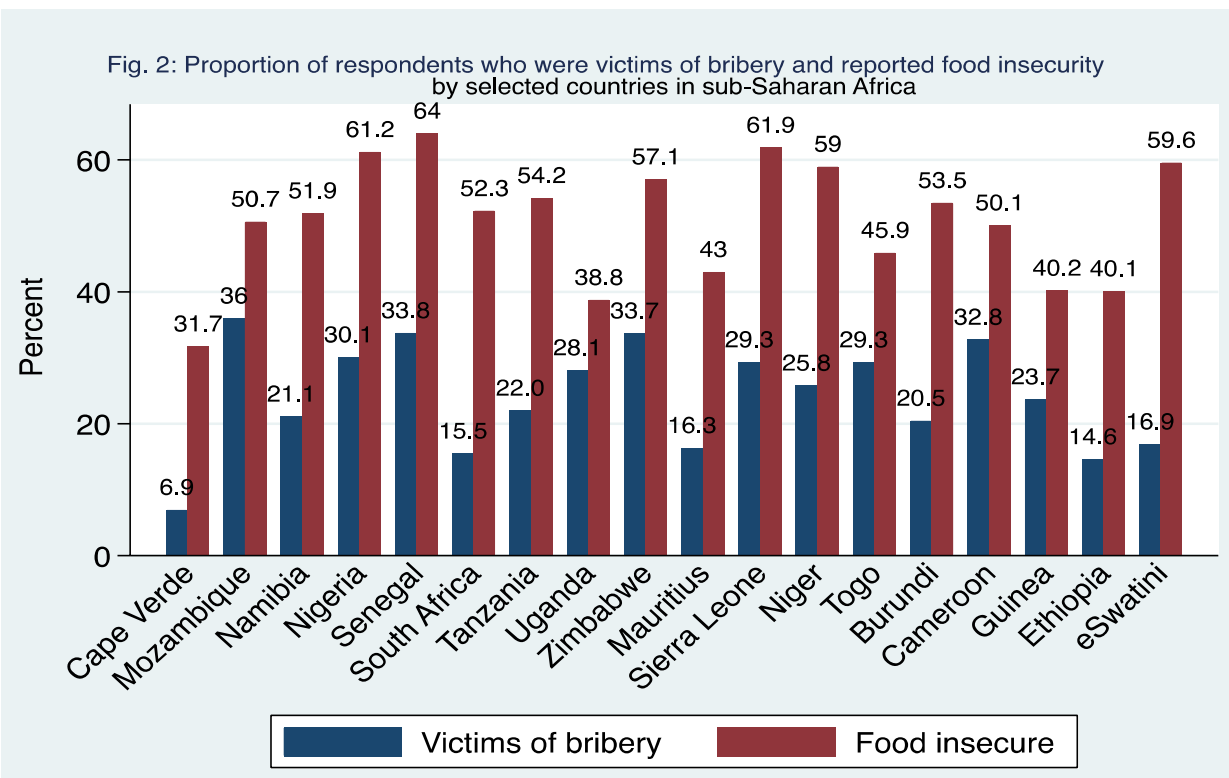

Source: Afrobarometer Survey - Rounds 5, 6, \& 7 
Table 1 Variable descriptions and summary statistics

\begin{tabular}{|c|c|c|c|c|}
\hline Variable & Description & Mean & S.D & Range \\
\hline Roadblock by police/army & Equal 1 if there is any roadblock set up by police or army in the area and zero otherwise & 0.064 & 0.245 & $0-1$ \\
\hline Food insecurity (binary) & $\begin{array}{l}\text { Equal to } 1 \text { if the respondent indicated "just once or twice, several times, or many time" to the } \\
\text { question" "Over the past year, how often, if ever, have you or anyone in your family: Gone } \\
\text { without enough food to eat"" and zero otherwise }\end{array}$ & 0.500 & 0.500 & $0-1$ \\
\hline Food insecurity (ordinal) & $\begin{array}{l}\text { Ordinal variable based on the question" "Over the past year, how often, if ever, have you or } \\
\text { anyone in your family: Gone without enough food to eat"" [1=Just once or twice, } 2=\text { Sev- } \\
\text { eral, } 3=\text { Many, } 4=\text { Always }]\end{array}$ & 2.060 & 0.875 & $1-4$ \\
\hline Just once or twice & $\begin{array}{l}\text { Equals to } 1 \text { if the respondent or someone in their household reported "just once or twice" } \\
\text { food insecurity; zero otherwise }\end{array}$ & 0.319 & 0.466 & $0-1$ \\
\hline Several times & $\begin{array}{l}\text { Equals to } 1 \text { if the respondent or someone in their household reported "several times" food } \\
\text { insecurity; zero otherwise }\end{array}$ & 0.174 & 0.379 & $0-1$ \\
\hline Many times & $\begin{array}{l}\text { Equals one if the respondent or someone in their household reported "many times" food } \\
\text { insecurity; zero otherwise }\end{array}$ & 0.152 & 0.359 & $0-1$ \\
\hline Always & $\begin{array}{l}\text { Equals to } 1 \text { if the respondent or someone in their household reported food insecurity; zero } \\
\text { otherwise }\end{array}$ & 0.068 & 0.252 & $0-1$ \\
\hline Bribery & $\begin{array}{l}\text { Equals to } 1 \text { if a respondent in the past one year reported to have paid a bribe before getting } \\
\text { a government ID, medical care in a public clinic, water and sanitation, police assistance, } \\
\text { assistance from the court, public school services, or avoid problems with police; zero } \\
\text { otherwise }\end{array}$ & 0.238 & 0.426 & $0-1$ \\
\hline Never & $\begin{array}{l}\text { Equals to } 1 \text { if the respondent reported to have never paid a bribe in the past year; zero other- } \\
\text { wise }\end{array}$ & 0.769 & 0.421 & $0-1$ \\
\hline Once or twice & $\begin{array}{l}\text { Equals to } 1 \text { if the respondent reported to have paid a bribe once or twice in the past year; } \\
\text { zero otherwise }\end{array}$ & 0.160 & 0.366 & $0-1$ \\
\hline A Few Times & $\begin{array}{l}\text { Equal to } 1 \text { if the respondent reported to have paid bribes a few times in the past year; zero } \\
\text { otherwise }\end{array}$ & 0.090 & 0.286 & $0-1$ \\
\hline Often & $\begin{array}{l}\text { Equal the one if the respondent reported to have paid bribes often in the past year; otherwise, } \\
\text { zero }\end{array}$ & 0.059 & 0.236 & $0-1$ \\
\hline No contact in the past year & Equal to 1 if the respondent reported to have no contact in the past year; zero otherwise & 0.814 & 0.389 & $0-1$ \\
\hline Female & Equals to 1 if female; zero otherwise & 0.500 & 0.500 & $0-1$ \\
\hline Urban & Equals to 1 if the respondent resided in an urban town; zero otherwise & 0.406 & 0.491 & $0-1$ \\
\hline Household size & Total number of adult citizens in the respondent's household & 3.738 & 2.649 & $1-59$ \\
\hline Education & $\begin{array}{l}\text { Respondent's highest educational qualification }[0=\text { No formal education, } 1=\text { Informal school } \\
\text { only, } 2=\text { Some primary schooling, } 3=\text { Primary school completed, } 4=\text { Some Secondary } \\
\text { school/high school, } 5=\text { Secondary school completed/high school, } 6=\text { post-secondary quali- } \\
\text { fication, not university, } 7=\text { Some university, } 8=\text { University completed, } 9=\text { post-graduate }]\end{array}$ & 3.334 & 2.129 & $0-9$ \\
\hline Age & $\begin{array}{l}\text { Condensed Respondent's age }[1=18-25 \text { years, } 2=26-35 \text { years, } 3=36-45 \text { years, } 4=46- \\
55 \text { years, } 6=\text { Over } 65 \text { years }] \\
=\end{array}$ & 2.634 & 1.437 & $1-6$ \\
\hline Asset & $\begin{array}{l}\text { Equals one if the respondent reported owning a radio, television, motor vehicle, car, motor- } \\
\text { cycle, or a mobile phone; zero otherwise }\end{array}$ & 0.832 & 0.374 & $0-1$ \\
\hline Community involvement & $\begin{array}{l}\text { A measure of respondent's community involvement based on the question" "Could you } \\
\text { tell me whether you are an official leader, an active member, an inactive member, or not } \\
\text { a member: Some other voluntary association or community group? }[0=\text { Not a member, } \\
1=\text { Inactive member, } 2=\text { Active member, } 3=\text { Official leader] }\end{array}$ & 0.684 & 0.970 & $0-3$ \\
\hline
\end{tabular}

with the country effect. Also, the latter model was fitted with an interaction term; however, both served as robustness checks. The average treatment effect (ATE) for models with both year and regional effects variable showed a negative and robust impact of the exposure to bribery on household food security status. Estimates of the variable of interest in the regressions with country effect and interaction term were negative and statistically significant. Although the likelihood-ratio test indicates that we cannot reject the null hypothesis of no correlation between treatment errors and the outcome errors, it was not surprising. Fischer (2010) affirmed that not controlling for region effect in a situation where there is a lack of sufficient variation in the variable of interest over time tends to cause severe bias. Models V and VIII tested the primary independent variable's binary configuration.

The principal finding in the logistic regressions is that victims of bribery were at least 30 percent likely to report 
Table 2 Regression results for the effect of bribery on household food security in Sub-Saharan Africa

\begin{tabular}{|c|c|c|c|c|c|c|c|c|}
\hline Variable & $\begin{array}{l}\text { EDVM } \\
\text { Model I }\end{array}$ & $\begin{array}{l}\text { EDVM } \\
\text { Model II }\end{array}$ & $\begin{array}{l}\text { EDVM } \\
\text { Model III }\end{array}$ & $\begin{array}{l}\text { EDMV } \\
\text { Model IV }\end{array}$ & $\begin{array}{l}\text { Logistic } \\
\text { Model V }\end{array}$ & $\begin{array}{l}\text { Logistic } \\
\text { Model VI }\end{array}$ & $\begin{array}{l}\text { Logistic } \\
\text { Model VII }\end{array}$ & $\begin{array}{l}\text { Logistic } \\
\text { Model VIII }\end{array}$ \\
\hline Constant & $\begin{array}{l}0.491 * * * \\
(0.013)\end{array}$ & $\begin{array}{l}0.499 * * * \\
(0.013)\end{array}$ & $\begin{array}{l}0.597 * * * \\
(0.018)\end{array}$ & $\begin{array}{l}0.602 * * * \\
(0.018)\end{array}$ & $\begin{array}{l}1.344 * * * \\
(0.059)\end{array}$ & $\begin{array}{l}1.339 * * * \\
(0.060)\end{array}$ & $\begin{array}{l}1.631 * * * \\
(0.098)\end{array}$ & $\begin{array}{l}1.644 * * * \\
(0.101)\end{array}$ \\
\hline Female & $\begin{array}{l}-0.012 * * * \\
(0.003)\end{array}$ & $\begin{array}{l}-0.012 * * * \\
(0.003)\end{array}$ & $\begin{array}{l}-0.011 * * * \\
(0.003)\end{array}$ & $\begin{array}{l}-0.011 * * * \\
(0.003)\end{array}$ & $\begin{array}{l}0.950 * * * \\
(0.012)\end{array}$ & $\begin{array}{l}0.949 * * * \\
(0.012)\end{array}$ & $\begin{array}{l}0.952 * * * \\
(0.012)\end{array}$ & $\begin{array}{l}0.952 * * * \\
(0.012)\end{array}$ \\
\hline Urban & $\begin{array}{l}-0.061 * * * \\
(0.003)\end{array}$ & $\begin{array}{l}-0.061 * * * \\
(0.003)\end{array}$ & $\begin{array}{l}-0.065 * * * \\
(0.003)\end{array}$ & $\begin{array}{l}-0.065^{* * * *} \\
(0.003)\end{array}$ & $\begin{array}{l}0.769 * * * \\
(0.010)\end{array}$ & $\begin{array}{l}0.767 * * * \\
(0.010)\end{array}$ & $\begin{array}{l}0.754 * * * \\
(0.010)\end{array}$ & $\begin{array}{l}0.753 * * * \\
(0.010)\end{array}$ \\
\hline Household size & $\begin{array}{l}0.003 * * * \\
(0.001)\end{array}$ & $\begin{array}{l}0.003 * * * \\
(0.001)\end{array}$ & $\begin{array}{l}-0.001 * * \\
(0.001)\end{array}$ & $\begin{array}{l}-0.001 * * \\
(0.001)\end{array}$ & $\begin{array}{l}1.012 * * * \\
(0.002)\end{array}$ & $\begin{array}{l}1.011 * * * \\
(0.002)\end{array}$ & $\begin{array}{l}0.994 * * \\
(0.002)\end{array}$ & $\begin{array}{l}0.994 * * \\
(0.002)\end{array}$ \\
\hline Asset & $\begin{array}{l}-0.109 * * * \\
(0.004)\end{array}$ & $\begin{array}{l}-0.122 * * * \\
(0.004)\end{array}$ & $\begin{array}{l}-0.111 * * * \\
(0.004)\end{array}$ & $\begin{array}{l}-0.120 * * * \\
(0.004)\end{array}$ & $\begin{array}{l}0.593 * * * \\
(0.012)\end{array}$ & $\begin{array}{l}0.620 * * * \\
(0.011)\end{array}$ & $\begin{array}{l}0.592 * * * \\
(0.012)\end{array}$ & $\begin{array}{l}0.607 * * * \\
(0.011)\end{array}$ \\
\hline Victim of bribery: at least once & $\begin{array}{l}0.413 * * * \\
(0.035)\end{array}$ & $\begin{array}{l}0.369 * * * \\
(0.037)\end{array}$ & $\begin{array}{l}0.143 * * * \\
(0.050)\end{array}$ & $\begin{array}{l}0.040 * * * \\
(0.009)\end{array}$ & $\begin{array}{l}1.304 * * * \\
(0.050)\end{array}$ & & $\begin{array}{l}1.350 * * * \\
(0.052)\end{array}$ & \\
\hline Victim of Bribery*Asset & & $\begin{array}{l}0.060 * * * \\
(0.009)\end{array}$ & & $\begin{array}{l}0.040 * * * \\
(0.009)\end{array}$ & $\begin{array}{l}1.268 * * * \\
(0.052)\end{array}$ & & $\begin{array}{l}1.159 * * * \\
(0.048)\end{array}$ & \\
\hline $\begin{array}{l}\text { Frequency of victim of bribery [ref: } \\
\text { never] } \\
\text { Once or twice }\end{array}$ & & & & & & $\begin{array}{l}1.384 * * * \\
(0.024)\end{array}$ & & $\begin{array}{l}1.339 * * * \\
(0.024)\end{array}$ \\
\hline A few times & & & & & & $\begin{array}{l}1.453 * * * \\
(0.033)\end{array}$ & & $\begin{array}{l}1.421 * * * \\
(0.033)\end{array}$ \\
\hline Often & & & & & & $\begin{array}{l}1.441 * * * \\
(0.039)\end{array}$ & & $\begin{array}{l}1.416 * * * \\
(0.039)\end{array}$ \\
\hline No contact in the past year & & & & & & $\begin{array}{l}0.947 * * * \\
(0.002)\end{array}$ & & $\begin{array}{l}0.974 \\
(0.019)\end{array}$ \\
\hline \multicolumn{9}{|l|}{ Age [ref: Over 65] } \\
\hline $18-25$ & $\begin{array}{l}-0.010 \\
(0.007)\end{array}$ & $\begin{array}{l}-0.010 \\
(0.007)\end{array}$ & $\begin{array}{l}-0.016 * * \\
(0.007)\end{array}$ & $\begin{array}{l}-0.016 * * \\
(0.007)\end{array}$ & $\begin{array}{l}0.960 \\
(0.029)\end{array}$ & $\begin{array}{l}0.964 \\
(0.030)\end{array}$ & $\begin{array}{l}0.930 * * * \\
(0.029)\end{array}$ & $\begin{array}{l}0.932 * * * \\
(0.029)\end{array}$ \\
\hline $26-35$ & $\begin{array}{l}0.040 * * * \\
(0.007)\end{array}$ & $\begin{array}{l}0.040 * * * \\
(0.007)\end{array}$ & $\begin{array}{l}0.030 * * * \\
(0.007)\end{array}$ & $\begin{array}{l}0.030 * * * \\
(0.007)\end{array}$ & $\begin{array}{l}1.191 * * * \\
(0.036)\end{array}$ & $\begin{array}{l}1.195 * * * \\
(0.036)\end{array}$ & $\begin{array}{l}1.142 * * * \\
(0.035)\end{array}$ & $\begin{array}{l}1.145^{* * * *} \\
(0.035)\end{array}$ \\
\hline $36-45$ & $\begin{array}{l}0.048 * * * \\
(0.007)\end{array}$ & $\begin{array}{l}0.049 * * * \\
(0.007)\end{array}$ & $\begin{array}{l}0.039 * * * \\
(0.007)\end{array}$ & $\begin{array}{l}0.039 * * * \\
(0.007)\end{array}$ & $\begin{array}{l}1.235 * * * \\
(0.038)\end{array}$ & $\begin{array}{l}1.236 * * * \\
(0.038)\end{array}$ & $\begin{array}{l}1.190 * * * \\
(0.037)\end{array}$ & $\begin{array}{l}1.190 * * * \\
(0.037)\end{array}$ \\
\hline $46-55$ & $\begin{array}{l}0.028 * * * \\
(0.007)\end{array}$ & $\begin{array}{l}0.028 * * * \\
(0.007)\end{array}$ & $\begin{array}{l}0.025 * * * \\
(0.007)\end{array}$ & $\begin{array}{l}0.025 * * * \\
(0.007)\end{array}$ & $\begin{array}{l}1.127 * * * \\
(0.036)\end{array}$ & $\begin{array}{l}1.130 * * * \\
(0.037)\end{array}$ & $\begin{array}{l}1.118 * * * \\
(0.037)\end{array}$ & $\begin{array}{l}1.120 * * * \\
(0.037)\end{array}$ \\
\hline $56-65$ & $\begin{array}{l}0.017 * * * \\
(0.008)\end{array}$ & $\begin{array}{l}0.018 * * \\
(0.008)\end{array}$ & $\begin{array}{l}0.019 * * \\
(0.008)\end{array}$ & $\begin{array}{l}0.019 * * \\
(0.008)\end{array}$ & $\begin{array}{l}1.080 * * \\
(0.037)\end{array}$ & $\begin{array}{l}1.080 * * \\
(0.037)\end{array}$ & $\begin{array}{l}1.086^{* * * *} \\
(0.038)\end{array}$ & $\begin{array}{l}1.086 * * \\
(0.038)\end{array}$ \\
\hline \multicolumn{9}{|l|}{ Employment [ref: No (not looking)] } \\
\hline No (looking) & $\begin{array}{l}0.058 * * * \\
(0.004)\end{array}$ & $\begin{array}{l}0.058 * * * \\
(0.004)\end{array}$ & $\begin{array}{l}0.053 * * * \\
(0.004)\end{array}$ & $\begin{array}{l}0.053 * * * \\
(0.004)\end{array}$ & $\begin{array}{l}1.286^{* * * *} \\
(0.020)\end{array}$ & $\begin{array}{l}1.281 * * * \\
(0.020)\end{array}$ & $\begin{array}{l}1.267 * * * \\
(0.021)\end{array}$ & $\begin{array}{l}1.264^{* * * *} \\
(0.020)\end{array}$ \\
\hline Yes, part-time & $\begin{array}{l}0.006 \\
(0.005)\end{array}$ & $\begin{array}{l}0.006 \\
(0.005)\end{array}$ & $\begin{array}{l}0.015 * * * \\
(0.005)\end{array}$ & $\begin{array}{l}0.015 * * * \\
(0.005)\end{array}$ & $\begin{array}{l}1.026 \\
(0.022)\end{array}$ & $\begin{array}{l}1.019 \\
(0.021)\end{array}$ & $\begin{array}{l}1.068 * * * \\
(0.023)\end{array}$ & $\begin{array}{l}1.063 * * * \\
(0.023)\end{array}$ \\
\hline Yes, full time & $\begin{array}{l}-0.104 * * * \\
(0.004)\end{array}$ & $\begin{array}{l}-0.104 * * * \\
(0.004)\end{array}$ & $\begin{array}{l}-0.088 * * * \\
(0.004)\end{array}$ & $\begin{array}{l}-0.088 * * * \\
(0.004)\end{array}$ & $\begin{array}{l}0.635^{* * * *} \\
(0.011)\end{array}$ & $\begin{array}{l}0.634 * * * \\
(0.011)\end{array}$ & $\begin{array}{l}0.675 * * * \\
(0.012)\end{array}$ & $\begin{array}{l}0.675 * * * \\
(0.012)\end{array}$ \\
\hline \multicolumn{9}{|l|}{ Education [ref: No formal schooling] } \\
\hline Informal schooling only & $\begin{array}{l}-0.031 * * * \\
(0.007)\end{array}$ & $\begin{array}{l}-0.031 * * * \\
(0.007)\end{array}$ & $\begin{array}{l}-0.033 * * * \\
(0.007)\end{array}$ & $\begin{array}{l}-0.033 * * * \\
(0.007)\end{array}$ & $\begin{array}{l}0.872 * * * \\
(0.027)\end{array}$ & $\begin{array}{l}0.871 * * * \\
(0.027)\end{array}$ & $\begin{array}{l}0.861 * * * \\
(0.027)\end{array}$ & $\begin{array}{l}0.863 * * * \\
(0.027)\end{array}$ \\
\hline Some primary schooling & $\begin{array}{l}-0.032 * * * \\
(0.005)\end{array}$ & $\begin{array}{l}-0.031 * * \\
(0.005)\end{array}$ & $\begin{array}{l}-0.009 * * * \\
(0.005)\end{array}$ & $\begin{array}{l}-0.009 * \\
(0.005)\end{array}$ & $\begin{array}{l}0.872 * * * \\
(0.020)\end{array}$ & $\begin{array}{l}0.870 * * * \\
(0.020)\end{array}$ & $\begin{array}{l}0.961 * \\
(0.022)\end{array}$ & $\begin{array}{l}0.961 * \\
(0.022)\end{array}$ \\
\hline Primary school completed & $\begin{array}{l}-0.103 * * * \\
(0.006)\end{array}$ & $\begin{array}{l}-0.102 * * * \\
(0.006)\end{array}$ & $\begin{array}{l}-0.073 * * * \\
(0.006)\end{array}$ & $\begin{array}{l}-0.722 * * * \\
(0.006)\end{array}$ & $\begin{array}{l}0.645 * * * \\
(0.016)\end{array}$ & $\begin{array}{l}0.641 * * * \\
(0.016)\end{array}$ & $\begin{array}{l}0.730 * * * \\
(0.018)\end{array}$ & $\begin{array}{l}0.728 * * * \\
(0.018)\end{array}$ \\
\hline Some secondary school/high school & $\begin{array}{l}-0.129 * * * \\
(0.005)\end{array}$ & $\begin{array}{l}-0.129 * * * \\
(0.005)\end{array}$ & $\begin{array}{l}-0.109 * * * \\
(0.005)\end{array}$ & $\begin{array}{l}-0.108 * * * \\
(0.005)\end{array}$ & $\begin{array}{l}0.576 * * * \\
(0.013)\end{array}$ & $\begin{array}{l}0.573 * * * \\
(0.013)\end{array}$ & $\begin{array}{l}0.623 * * * \\
(0.014)\end{array}$ & $\begin{array}{l}0.622 * * * \\
(0.014)\end{array}$ \\
\hline secondary school completed/high school & $\begin{array}{l}-0.178 * * * \\
(0.006)\end{array}$ & $\begin{array}{l}-0.178 * * * \\
(0.006)\end{array}$ & $\begin{array}{l}-0.161^{* * * *} \\
(0.006)\end{array}$ & $\begin{array}{l}-0.161^{* * *} \\
(0.006)\end{array}$ & $\begin{array}{l}0.468 * * * \\
(0.012)\end{array}$ & $\begin{array}{l}0.463^{* * * *} \\
(0.011)\end{array}$ & $\begin{array}{l}0.497 * * * \\
(0.012)\end{array}$ & $\begin{array}{l}0.494 * * * \\
(0.012)\end{array}$ \\
\hline Post-secondary qualifications, not univ & $\begin{array}{l}-0.233 * * * \\
(0.007)\end{array}$ & $\begin{array}{l}-0.233 * * * \\
(0.007)\end{array}$ & $\begin{array}{l}-0.215^{* * * *} \\
(0.007)\end{array}$ & $\begin{array}{l}-0.215^{* * * *} \\
(0.007)\end{array}$ & $\begin{array}{l}0.364 * * * \\
(0.012)\end{array}$ & $\begin{array}{l}0.361 * * * \\
(0.012)\end{array}$ & $\begin{array}{l}0.388 * * * \\
(0.013)\end{array}$ & $\begin{array}{l}0.386 * * * \\
(0.013)\end{array}$ \\
\hline
\end{tabular}


Table 2 (continued)

\begin{tabular}{|c|c|c|c|c|c|c|c|c|}
\hline Variable & $\begin{array}{l}\text { EDVM } \\
\text { Model I }\end{array}$ & $\begin{array}{l}\text { EDVM } \\
\text { Model II }\end{array}$ & $\begin{array}{l}\text { EDVM } \\
\text { Model III }\end{array}$ & $\begin{array}{l}\text { EDMV } \\
\text { Model IV }\end{array}$ & $\begin{array}{l}\text { Logistic } \\
\text { Model V }\end{array}$ & $\begin{array}{l}\text { Logistic } \\
\text { Model VI }\end{array}$ & $\begin{array}{l}\text { Logistic } \\
\text { Model VII }\end{array}$ & $\begin{array}{l}\text { Logistic } \\
\text { Model VIII }\end{array}$ \\
\hline Some university & $\begin{array}{l}-0.195 * * * \\
(0.009)\end{array}$ & $\begin{array}{l}-0.195 * * * \\
(0.009)\end{array}$ & $\begin{array}{l}-0.187 * * * \\
(0.009)\end{array}$ & $\begin{array}{l}-0.187 * * * \\
(0.009)\end{array}$ & $\begin{array}{l}0.432 * * * \\
(0.017)\end{array}$ & $\begin{array}{l}0.429 * * * \\
(0.017)\end{array}$ & $\begin{array}{l}0.440 * * * \\
(0.017)\end{array}$ & $\begin{array}{l}0.439 * * * \\
(0.017)\end{array}$ \\
\hline University completed & $\begin{array}{l}-0.261 * * * \\
(0.009)\end{array}$ & $\begin{array}{l}-0.261 * * * \\
(0.008)\end{array}$ & $\begin{array}{l}-0.249 * * * \\
(0.008)\end{array}$ & $\begin{array}{l}-0.249 * * * \\
(0.008)\end{array}$ & $\begin{array}{l}0.312 * * * \\
(0.013)\end{array}$ & $\begin{array}{l}0.309 * * * \\
(0.012)\end{array}$ & $\begin{array}{l}0.322 * * * \\
(0.013)\end{array}$ & $\begin{array}{l}0.319 * * * \\
(0.013)\end{array}$ \\
\hline Post-graduate & $\begin{array}{l}-0.337 * * * \\
(0.014)\end{array}$ & $\begin{array}{l}-0.337 * * * \\
(0.014)\end{array}$ & $\begin{array}{l}-0.307 * * * \\
(0.015)\end{array}$ & $\begin{array}{l}-0.307 * * * \\
(0.015)\end{array}$ & $\begin{array}{l}0.198 * * * \\
(0.019)\end{array}$ & $\begin{array}{l}0.196 * * * \\
(0.019)\end{array}$ & $\begin{array}{l}0.218 * * * \\
(0.021)\end{array}$ & $\begin{array}{l}0.217 * * * \\
(0.021)\end{array}$ \\
\hline \multicolumn{9}{|c|}{$\begin{array}{l}\text { Community membership [Ref: Not a } \\
\text { member] }\end{array}$} \\
\hline Inactive member & $\begin{array}{l}0.042 * * * \\
(0.004)\end{array}$ & $\begin{array}{l}0.042 * * * \\
(0.004)\end{array}$ & $\begin{array}{l}0.034 * * * \\
(0.004)\end{array}$ & $\begin{array}{l}0.034 * * * \\
(0.004)\end{array}$ & $\begin{array}{l}1.202 * * * \\
(0.022)\end{array}$ & $\begin{array}{l}1.196 * * * \\
(0.022)\end{array}$ & $\begin{array}{l}1.159 * * * \\
(0.022)\end{array}$ & $\begin{array}{l}1.154 * * * \\
(0.022)\end{array}$ \\
\hline Active member & $\begin{array}{l}0.025 * * * \\
(0.004)\end{array}$ & $\begin{array}{l}0.025 * * * \\
(0.004\end{array}$ & $\begin{array}{l}0.017 * * * \\
(0.004)\end{array}$ & $\begin{array}{l}0.017 * * * \\
(0.004)\end{array}$ & $\begin{array}{l}1.116^{* * * *} \\
(0.018)\end{array}$ & $\begin{array}{l}1.106^{* * * *} \\
(0.018)\end{array}$ & $\begin{array}{l}1.082 * * * \\
(0.018)\end{array}$ & $\begin{array}{l}1.074 * * * \\
(0.018)\end{array}$ \\
\hline Official member & $\begin{array}{l}0.031 * * * \\
(0.006)\end{array}$ & $\begin{array}{l}0.031 * * * \\
(0.006)\end{array}$ & $\begin{array}{l}0.019 * * * \\
(0.006)\end{array}$ & $\begin{array}{l}0.019 * * * \\
(0.006)\end{array}$ & $\begin{array}{l}1.147 * * * \\
(0.031)\end{array}$ & $\begin{array}{l}1.138 * * * \\
(0.050)\end{array}$ & $\begin{array}{l}1.089 * * * \\
(0.030)\end{array}$ & $\begin{array}{l}1.081 * * * \\
(0.029)\end{array}$ \\
\hline Year effect & Yes & Yes & Yes & Yes & Yes & Yes & Yes & Yes \\
\hline Region effect & Yes & Yes & Yes & Yes & Yes & Yes & Yes & Yes \\
\hline Country effect & No & No & No & No & No & No & No & No \\
\hline Pseudo $\mathrm{R}^{2}$ & & & & & 0.069 & 0.069 & 0.082 & 0.082 \\
\hline LR chi $^{2}$ & & & & & $11,210.24$ & $11,343.47$ & $13,423.41$ & $13,423.41$ \\
\hline Log-Likelihood & & & & & $-76,247.81$ & $-76,181.20$ & $-75,141.23$ & $75,141.23$ \\
\hline Wald chi ${ }^{2}$ & $13,057.33$ & $13,119.54$ & $16,703.00$ & $16,741.21$ & & & & \\
\hline Wald test $($ rho $=0)$ & $88.88 * * *$ & $81.15 * * *$ & 0.79 & 0.75 & & & & \\
\hline Log pseudolikelihood & $-144,665.3$ & $-144,642.2$ & $-143,597.8$ & $-143,587.6$ & & & & \\
\hline Number of observations & 118,089 & 118,089 & 118,809 & 118,809 & 118,089 & 118,089 & 118,089 & 118,089 \\
\hline
\end{tabular}

Estimates for logistic regressions are in odds ratios

$O R$ robust standard errors are in parentheses

*Significant at the 0.10 level

** Significant at the 0.05 level

${ }^{* * * *}$ Significant at the 0.01 level

one form of food insecurity than non-bribe paying families: thus, suggesting that bribery siphon resources from household food spending or budget, which, in turn, forces the affected household to ration or even forgo spending on food (Hunt \& Laszlo, 2012). The interaction term was positive and statistically significant, thus indicating respondents that owned assets were more eager to pay bribes. As soon as public employees realized the willingness, they exploited the gap (Krishna, 2007).

Further analysis of the primary independent variable's multiclass configuration revealed a 38 percent, 45 percent, and 44 percent likelihood of being food insecure for families that bribed once or twice a few times, and often, respectively. However, households that reported no contact in the past year were five percent less likely to experience food insecurity. Models VII and VIII served as the robustness checks. Notwithstanding, the exclusion of the region-effect resulted in estimates that were remarkably consistent with the baselines. They showed that victims of bribery were significantly more likely to experience food insecurity than their counterparts that never bribed or had no contact in the intervening period. In the same vein, as bribery incidence increases, so does the probability of being food insecure for respondents on the supply side. Specifically, respondents who paid bribes frequently, few times, and just once or twice were 34 percent, 42 percent, and 42 percent more likely to be food insecure than those that never paid. However, odds were lower for respondents who were not victims of bribery in the past 12 months.

All the findings relating to the control variables align with existing literature. For instance, a larger household was susceptible to the risk of food insecurity, and so were active and inactive involvement in voluntary community organizations, a proxy for social capital (Sseguya et al., 2018). The yeareffect revealed that food insecurity grew worse over time, and none of the regions was a haven for households. On the flip side, residency in urban areas, being a female, and owning assets (Akerele et al., 2013) mitigated the possibility of 
Table 3 Logistic regression effect of bribery via different channels on food-insecure respondents in Sub-Saharan Africa

\begin{tabular}{|c|c|c|c|c|c|c|c|c|}
\hline \multirow[t]{3}{*}{ Variable } & \multicolumn{8}{|c|}{ Sources of bribery } \\
\hline & \multicolumn{2}{|c|}{ Document or permit } & \multicolumn{2}{|c|}{ Treatment at the clinic } & \multicolumn{2}{|l|}{ Avoid police } & \multicolumn{2}{|c|}{ School placement } \\
\hline & Model IX & Model X & Model XI & Model XII & Model XIII & $\overline{\text { Model XIV }}$ & Model XV & Model XVI \\
\hline Constant & $\begin{array}{l}1.319 * * * \\
(0.057)\end{array}$ & $\begin{array}{l}1.339 * * * \\
(0.059)\end{array}$ & $\begin{array}{l}1.310 * * * \\
(0.056)\end{array}$ & $\begin{array}{l}1.341 * * * \\
(0.058)\end{array}$ & $\begin{array}{l}1.322 * * * \\
(0.057)\end{array}$ & $\begin{array}{l}1.366^{* * * *} \\
(0.060)\end{array}$ & $\begin{array}{l}1.339 * * * \\
(0.058)\end{array}$ & $\begin{array}{l}1.412 * * * \\
(0.062)\end{array}$ \\
\hline Female & $\begin{array}{l}0.943 * * * \\
(0.011)\end{array}$ & $\begin{array}{l}0.945 * * * \\
(0.012)\end{array}$ & $\begin{array}{l}0.930 * * * \\
(0.012)\end{array}$ & $\begin{array}{l}0.928 * * * \\
(0.012)\end{array}$ & $\begin{array}{l}0.946 * * * \\
(0.012)\end{array}$ & $\begin{array}{l}0.948 * * * \\
(0.012)\end{array}$ & $\begin{array}{l}0.931 * * * \\
(0.012)\end{array}$ & $\begin{array}{l}0.931 * * * \\
(0.012)\end{array}$ \\
\hline Urban & $\begin{array}{l}0.771 * * * \\
(0.010)\end{array}$ & $\begin{array}{l}0.770 * * * \\
(0.010)\end{array}$ & $\begin{array}{l}0.770 * * * \\
(0.010)\end{array}$ & $\begin{array}{l}0.771 * * * \\
(0.010)\end{array}$ & $\begin{array}{l}0.773 * * * \\
(0.010)\end{array}$ & $\begin{array}{l}0.772^{* * * *} \\
(0.010)\end{array}$ & $\begin{array}{l}0.771 * * * \\
(0.010)\end{array}$ & $\begin{array}{l}0.773 * * * \\
(0.010)\end{array}$ \\
\hline Household size & $\begin{array}{l}1.013 * * * \\
(0.002)\end{array}$ & $\begin{array}{l}1.013 * * * \\
(0.002)\end{array}$ & $\begin{array}{l}1.012 * * * \\
(0.002)\end{array}$ & $\begin{array}{l}1.012 * * * \\
(0.002)\end{array}$ & $\begin{array}{l}1.013 * * * \\
(0.002)\end{array}$ & $\begin{array}{l}1.013 * * * \\
(0.002)\end{array}$ & $\begin{array}{l}1.013 * * * \\
(0.002)\end{array}$ & $\begin{array}{l}1.012 * * * \\
(0.002)\end{array}$ \\
\hline Asset & $\begin{array}{l}0.623 * * * \\
(0.011)\end{array}$ & $\begin{array}{l}0.622 * * * \\
(0.011)\end{array}$ & $\begin{array}{l}0.627 * * * \\
(0.011)\end{array}$ & $\begin{array}{l}0.626 * * * \\
(0.011)\end{array}$ & $\begin{array}{l}0.624 * * * \\
(0.011)\end{array}$ & $\begin{array}{l}0.623 * * * \\
(0.011)\end{array}$ & $\begin{array}{l}0.627 * * * \\
(0.011)\end{array}$ & $\begin{array}{l}0.625 * * * \\
(0.011)\end{array}$ \\
\hline Bribery: at least once & $\begin{array}{l}1.682 * * * \\
(0.035)\end{array}$ & & $\begin{array}{l}1.857 * * * \\
(0.040)\end{array}$ & & $\begin{array}{l}1.584 * * * \\
(0.034)\end{array}$ & & $\begin{array}{l}1.835 * * * \\
(0.045)\end{array}$ & \\
\hline \multicolumn{9}{|l|}{ Frequency of bribery [ref: never] } \\
\hline Once or twice & & $\begin{array}{l}1.478 * * * \\
(0.040)\end{array}$ & & $\begin{array}{l}1.675^{* * * *} \\
(0.050)\end{array}$ & & $\begin{array}{l}1.428 * * * \\
(0.045)\end{array}$ & & $\begin{array}{l}1.697 * * * \\
(0.057)\end{array}$ \\
\hline A few times & & $\begin{array}{l}1.902 * * * \\
(0.075)\end{array}$ & & $\begin{array}{l}1.962 * * * \\
(0.073)\end{array}$ & & $\begin{array}{l}1.628 * * * \\
(0.063)\end{array}$ & & $\begin{array}{l}1.845^{* * * *} \\
(0.083)\end{array}$ \\
\hline Often & & $\begin{array}{l}2.035^{* * * *} \\
(0.102)\end{array}$ & & $\begin{array}{l}1.982 * * * \\
(0.091)\end{array}$ & & $\begin{array}{l}1.631 * * * \\
(0.066)\end{array}$ & & $\begin{array}{l}1.772 * * * \\
(0.100)\end{array}$ \\
\hline No contact in the past year & & $\begin{array}{l}0.968^{*} \\
(0.013)\end{array}$ & & $\begin{array}{l}0.938 * * * \\
(0.013)\end{array}$ & & $\begin{array}{l}0.944 * * * \\
(0.014)\end{array}$ & & $\begin{array}{l}0.903 * * * \\
(0.012)\end{array}$ \\
\hline \multicolumn{9}{|l|}{ Age [ref: Over 65] } \\
\hline $18-25$ & $\begin{array}{l}0.981 \\
(0.030)\end{array}$ & $\begin{array}{l}0.981 \\
(0.030)\end{array}$ & $\begin{array}{l}0.983 \\
(0.030)\end{array}$ & $\begin{array}{l}0.985 \\
(0.030)\end{array}$ & $\begin{array}{l}0.984 \\
(0.030)\end{array}$ & $\begin{array}{l}0.983 \\
(0.030)\end{array}$ & $\begin{array}{l}0.981 \\
(0.030)\end{array}$ & $\begin{array}{l}0.976 \\
(0.030)\end{array}$ \\
\hline $26-35$ & $\begin{array}{l}1.222 * * * \\
(0.037)\end{array}$ & $\begin{array}{l}1.220 * * * \\
(0.037)\end{array}$ & $\begin{array}{l}1.225 * * * \\
(0.037)\end{array}$ & $\begin{array}{l}1.224 * * * \\
(0.037)\end{array}$ & $\begin{array}{l}1.221 * * * \\
(0.037)\end{array}$ & $\begin{array}{l}1.220 * * * \\
(0.037)\end{array}$ & $\begin{array}{l}1.227 * * * \\
(0.037)\end{array}$ & $\begin{array}{l}1.216 * * * \\
(0.037)\end{array}$ \\
\hline $36-45$ & $\begin{array}{l}1.260 * * * \\
(0.039)\end{array}$ & $\begin{array}{l}1.258 * * * \\
(0.039)\end{array}$ & $\begin{array}{l}1.262 * * * \\
(0.039)\end{array}$ & $\begin{array}{l}1.261^{* * * *} \\
(0.039)\end{array}$ & $\begin{array}{l}1.259 * * * \\
(0.039)\end{array}$ & $\begin{array}{l}1.256 * * * \\
(0.039)\end{array}$ & $\begin{array}{l}1.258 * * * \\
(0.039)\end{array}$ & $\begin{array}{l}1.243 * * * \\
(0.038)\end{array}$ \\
\hline $46-55$ & $\begin{array}{l}1.144 * * * \\
(0.037)\end{array}$ & $\begin{array}{l}1.142 * * * \\
(0.037)\end{array}$ & $\begin{array}{l}1.144 * * * \\
(0.037)\end{array}$ & $\begin{array}{l}1.144 * * * \\
(0.037)\end{array}$ & $\begin{array}{l}1.144 * * * \\
(0.037)\end{array}$ & $\begin{array}{l}1.143 * * * \\
(0.037)\end{array}$ & $\begin{array}{l}1.145 * * * \\
(0.037)\end{array}$ & $\begin{array}{l}1.132 * * * \\
(0.037)\end{array}$ \\
\hline $56-65$ & $\begin{array}{l}1.087 * * * \\
(0.037)\end{array}$ & $\begin{array}{l}1.086 * * * \\
(0.037)\end{array}$ & $\begin{array}{l}1.086 * * \\
(0.037)\end{array}$ & $\begin{array}{l}1.086 * * * \\
(0.037)\end{array}$ & $\begin{array}{l}1.088^{* * * *} \\
(0.038)\end{array}$ & $\begin{array}{l}1.087 * * * \\
(0.037)\end{array}$ & $\begin{array}{l}1.085^{* * * *} \\
(0.037)\end{array}$ & $\begin{array}{l}1.079 * * \\
(0.036)\end{array}$ \\
\hline \multicolumn{9}{|l|}{ Employment [ref: No (not looking)] } \\
\hline No (looking) & $\begin{array}{l}1.289 * * * \\
(0.020)\end{array}$ & $\begin{array}{l}1.287 * * * \\
(0.020)\end{array}$ & $\begin{array}{l}1.288^{* * * *} \\
(0.020)\end{array}$ & $\begin{array}{l}1.287 * * * \\
(0.020)\end{array}$ & $\begin{array}{l}1.290 * * * \\
(0.020)\end{array}$ & $\begin{array}{l}1.288^{* * * *} \\
(0.020)\end{array}$ & $\begin{array}{l}1.294 * * * \\
(0.021)\end{array}$ & $\begin{array}{l}1.292 * * * \\
(0.020)\end{array}$ \\
\hline Yes, part-time & $\begin{array}{l}1.031 * * \\
(0.022)\end{array}$ & $\begin{array}{l}1.030 \\
(0.022)\end{array}$ & $\begin{array}{l}1.035 \\
(0.022)\end{array}$ & $\begin{array}{l}1.034 * * * \\
(0.022)\end{array}$ & $\begin{array}{l}1.034 \\
(0.022)\end{array}$ & $\begin{array}{l}1.033 \\
(0.022)\end{array}$ & $\begin{array}{l}1.040 * \\
(0.021)\end{array}$ & $\begin{array}{l}1.037 * \\
(0.022)\end{array}$ \\
\hline Yes, full time & $\begin{array}{l}0.636 * * * \\
(0.011)\end{array}$ & $\begin{array}{l}0.638 * * * \\
(0.011)\end{array}$ & $\begin{array}{l}0.640 * * * \\
(0.011)\end{array}$ & $\begin{array}{l}0.640 * * * \\
(0.011)\end{array}$ & $\begin{array}{l}0.635^{* * * *} \\
(0.011)\end{array}$ & $\begin{array}{l}0.634 * * * \\
(0.011)\end{array}$ & $\begin{array}{l}0.641 * * * \\
(0.011)\end{array}$ & $\begin{array}{l}0.641^{* * * *} \\
(0.011)\end{array}$ \\
\hline \multicolumn{9}{|l|}{ Education [ref: No formal schooling] } \\
\hline Informal schooling only & $\begin{array}{l}0.867 * * * \\
(0.027)\end{array}$ & $\begin{array}{l}0.866 * * * \\
(0.027)\end{array}$ & $\begin{array}{l}0.874 * * * \\
(0.027)\end{array}$ & $\begin{array}{l}0.871 * * * \\
(0.027)\end{array}$ & $\begin{array}{l}0.869 * * * \\
(0.027)\end{array}$ & $\begin{array}{l}0.868 * * * \\
(0.027)\end{array}$ & $\begin{array}{l}0.870 * * * \\
(0.027)\end{array}$ & $\begin{array}{l}0.866 * * * \\
(0.027)\end{array}$ \\
\hline Some primary schooling & $\begin{array}{l}0.878 * * * \\
(0.020)\end{array}$ & $\begin{array}{l}0.876 * * * \\
(0.020)\end{array}$ & $\begin{array}{l}0.876^{* * * *} \\
(0.020)\end{array}$ & $\begin{array}{l}0.876 * * * \\
(0.020)\end{array}$ & $\begin{array}{l}0.882 * * * \\
(0.020)\end{array}$ & $\begin{array}{l}0.881 * * * \\
(0.020)\end{array}$ & $\begin{array}{l}0.879 * * * \\
(0.020)\end{array}$ & $\begin{array}{l}0.878 * * * \\
(0.020)\end{array}$ \\
\hline Primary school completed & $\begin{array}{l}0.647 * * * \\
(0.016)\end{array}$ & $\begin{array}{l}0.645 * * * \\
(0.016)\end{array}$ & $\begin{array}{l}0.649 * * * \\
(0.016)\end{array}$ & $\begin{array}{l}0.648 * * * \\
(0.016)\end{array}$ & $\begin{array}{l}0.650 * * * \\
(0.016)\end{array}$ & $\begin{array}{l}0.649 * * * \\
(0.016)\end{array}$ & $\begin{array}{l}0.649 * * * \\
(0.016)\end{array}$ & $\begin{array}{l}0.646 * * * \\
(0.016)\end{array}$ \\
\hline Some secondary school/high school & $\begin{array}{l}0.578 * * * \\
(0.013)\end{array}$ & $\begin{array}{l}0.576 * * * \\
(0.013)\end{array}$ & $\begin{array}{l}0.583 * * * \\
(0.013)\end{array}$ & $\begin{array}{l}0.583 * * * \\
(0.013)\end{array}$ & $\begin{array}{l}0.585^{* * * *} \\
(0.013)\end{array}$ & $\begin{array}{l}0.583 * * * \\
(0.013)\end{array}$ & $\begin{array}{l}0.582 * * * \\
(0.013)\end{array}$ & $\begin{array}{l}0.579 * * * \\
(0.013)\end{array}$ \\
\hline Secondary school completed/high school & $\begin{array}{l}0.471 * * * \\
(0.012)\end{array}$ & $\begin{array}{l}0.469 * * * \\
(0.012)\end{array}$ & $\begin{array}{l}0.478 * * * \\
(0.012)\end{array}$ & $\begin{array}{l}0.479 * * * \\
(0.012)\end{array}$ & $\begin{array}{l}0.476 * * * \\
(0.012)\end{array}$ & $\begin{array}{l}0.473 * * * \\
(0.012)\end{array}$ & $\begin{array}{l}0.478 * * * \\
(0.012)\end{array}$ & $\begin{array}{l}0.475 * * * \\
(0.012)\end{array}$ \\
\hline Post-secondary qualifications, not univ & $\begin{array}{l}0.368^{* * * *} \\
(0.012)\end{array}$ & $\begin{array}{l}0.366^{* * * *} \\
(0.012)\end{array}$ & $\begin{array}{l}0.380 * * * \\
(0.013)\end{array}$ & $\begin{array}{l}0.380 * * * \\
(0.013)\end{array}$ & $\begin{array}{l}0.373 * * * \\
(0.012)\end{array}$ & $\begin{array}{l}0.370 * * * \\
(0.012)\end{array}$ & $\begin{array}{l}0.379 * * * \\
(0.013)\end{array}$ & $\begin{array}{l}0.376^{* * *} * \\
(0.012)\end{array}$ \\
\hline
\end{tabular}


Table 3 (continued)

\begin{tabular}{|c|c|c|c|c|c|c|c|c|}
\hline \multirow[t]{3}{*}{ Variable } & \multicolumn{8}{|c|}{ Sources of bribery } \\
\hline & \multicolumn{2}{|c|}{ Document or permit } & \multicolumn{2}{|c|}{ Treatment at the clinic } & \multicolumn{2}{|l|}{ Avoid police } & \multicolumn{2}{|c|}{ School placement } \\
\hline & Model IX & Model X & Model XI & Model XII & Model XIII & Model XIV & Model XV & Model XVI \\
\hline Some university & $\begin{array}{l}0.434 * * * \\
(0.017)\end{array}$ & $\begin{array}{l}0.431 * * * \\
(0.017)\end{array}$ & $\begin{array}{l}0.445 * * * \\
(0.017)\end{array}$ & $\begin{array}{l}0.445^{* * *} \\
(0.017)\end{array}$ & $\begin{array}{l}0.444 * * * \\
(0.017)\end{array}$ & $\begin{array}{l}0.442 * * * \\
(0.017)\end{array}$ & $\begin{array}{l}0.445 * * * \\
(0.017)\end{array}$ & $\begin{array}{l}0.441 * * * \\
(0.017)\end{array}$ \\
\hline University completed & $\begin{array}{l}0.316 * * * \\
(0.013)\end{array}$ & $\begin{array}{l}0.313 * * * \\
(0.013)\end{array}$ & $\begin{array}{l}0.326 * * * \\
(0.013)\end{array}$ & $\begin{array}{l}0.326 * * * \\
(0.013)\end{array}$ & $\begin{array}{l}0.320 * * * \\
(0.013)\end{array}$ & $\begin{array}{l}0.317 * * * \\
(0.013)\end{array}$ & $\begin{array}{l}0.326 * * * \\
(0.013)\end{array}$ & $\begin{array}{l}0.323 * * * \\
(0.013)\end{array}$ \\
\hline Post-graduate & $\begin{array}{l}0.198 * * * \\
(0.019)\end{array}$ & $\begin{array}{l}0.196 * * * \\
(0.019)\end{array}$ & $\begin{array}{l}0.205^{* * *} \\
(0.020)\end{array}$ & $\begin{array}{l}0.206^{* * *} \\
(0.020)\end{array}$ & $\begin{array}{l}0.202 * * * \\
(0.019)\end{array}$ & $\begin{array}{l}0.200 * * * \\
(0.019)\end{array}$ & $\begin{array}{l}0.207 * * * \\
(0.020)\end{array}$ & $\begin{array}{l}0.206 * * * \\
(0.020)\end{array}$ \\
\hline \multicolumn{9}{|c|}{$\begin{array}{l}\text { Community membership [Ref: Not a } \\
\text { member] }\end{array}$} \\
\hline Inactive member & $\begin{array}{l}1.216 * * * \\
(0.023)\end{array}$ & $\begin{array}{l}1.214 * * * \\
(0.022)\end{array}$ & $\begin{array}{l}1.214 * * * \\
(0.022)\end{array}$ & $\begin{array}{l}1.212 * * * \\
(0.022)\end{array}$ & $\begin{array}{l}1.221 * * * \\
(0.023)\end{array}$ & $\begin{array}{l}1.219 * * * \\
(0.023)\end{array}$ & $\begin{array}{l}1.216 * * * \\
(0.023)\end{array}$ & $\begin{array}{l}1.209 * * * \\
(0.022)\end{array}$ \\
\hline Active member & $\begin{array}{l}1.134 * * * \\
(0.019)\end{array}$ & $\begin{array}{l}1.130 * * * \\
(0.019)\end{array}$ & $\begin{array}{l}1.127 * * * \\
(0.018)\end{array}$ & $\begin{array}{l}1.124 * * * \\
(0.019)\end{array}$ & $\begin{array}{l}1.135 * * * \\
(0.019)\end{array}$ & $\begin{array}{l}1.131 * * * \\
(0.019)\end{array}$ & $\begin{array}{l}1.128 * * * \\
(0.019)\end{array}$ & $\begin{array}{l}1.120 * * * \\
(0.019)\end{array}$ \\
\hline Official member & $\begin{array}{l}1.163 * * * \\
(0.031)\end{array}$ & $\begin{array}{l}1.159 * * * \\
(0.031)\end{array}$ & $\begin{array}{l}1.169 * * * \\
(0.031)\end{array}$ & $\begin{array}{l}1.163 * * * \\
(0.031)\end{array}$ & $\begin{array}{l}1.173 * * * \\
(0.032)\end{array}$ & $\begin{array}{l}1.170 \text { *** } \\
(0.031)\end{array}$ & $\begin{array}{l}1.164 * * * \\
(0.031)\end{array}$ & $\begin{array}{l}1.152 * * * \\
(0.031)\end{array}$ \\
\hline Year effect & Yes & Yes & Yes & Yes & Yes & Yes & Yes & Yes \\
\hline Region effect & Yes & Yes & Yes & Yes & Yes & Yes & Yes & Yes \\
\hline Pseudo $\mathrm{R}^{2}$ & 0.066 & 0.067 & 0.068 & 0.068 & 0.065 & 0.065 & 0.066 & 0.066 \\
\hline $\mathrm{LR} \mathrm{Chi}^{2}$ & $10,839.33$ & $10,897.66$ & $11,055.96$ & $11,094.06$ & $10,666.06$ & $10,692.22$ & $10,821.88$ & $10,882.21$ \\
\hline Log-likelihood & $-76,433.27$ & $-76,404$ & $-76,324.9$ & $-83,255.5$ & $-76,519.9$ & $-76,506.82$ & $-76,441.99$ & $-76,411.83$ \\
\hline Observations & 118,089 & 118,089 & 118,089 & 118,089 & 118,089 & 118,089 & 118,089 & 118,089 \\
\hline Rounds & $5,6, \& 7$ & $5,6, \& 7$ & $5,6, \& 7$ & $5,6, \& 7$ & $5,6, \& 7$ & $5,6, \& 7$ & $5,6, \& 7$ & $5,6, \& 7$ \\
\hline
\end{tabular}

Estimates for logistic regressions are in odds ratios

$O R$ robust standard errors are in parentheses

*Significant at the 0.10 level

** Significant at the 0.05 level

${ }^{* * * *}$ Significant at the 0.01 level

being food insecure. In addition, higher educational attainment offered a protective effect (Akerele et al., 2013) and, likewise, full-time employment (Etana \& Tolossa, 2017).

Table 3 captures how each government institution affects the food security status of bribe-paying respondents. The results revealed that any contact with any of the government institutions considered in the study significantly contributed to the probability of food insecurity. Each time a bribe is offered in exchange for an identity card, public school services, medical care in a local clinic, or to avoid a problem with the police, the food security status spiral downward. However, the condition worsens with increased incidences. In real terms, respondents that frequently bribe government officials were at least 63 percent more likely to report being food insecure than their similarly-situated respondents with no contact ever. The result demonstrates the weak structure of public institutions in Sub-Saharan Africa. Moreover, it serves as a clarion call for a paradigm shift in how businesses are conducted in government agencies and parastatals. Otherwise, the lasting solution to food insecurity might remain elusive in the region.
Lastly, ordered logistic regressions were carried out to underscore how the vulnerability created by exposure to bribery stack up across different gradients of food insecurity. Regardless of whether the primary variable of interest assumed a binary or ordinal construct, any level of exposure to bribery worsened respondents' food security status. The finding from the binary representation of the leading independent variable of interest indicated that respondents who were victims of bribery once or twice were at least 21 percent more likely to experience food insecurity than respondents who never paid bribes (see Table 4). Furthermore, the outlook worsened as the frequency of bribery increased. For instance, respondents who were victims of bribery a few times and frequently were 32 percent and 58 percent more likely to experience food insecurity, respectively. The finding is consistent with Seligson (2006), which opined that the consequence of corruption varies across people. Therefore, it can be surmised that the region's goal of becoming food secure depends on various governments' willingness to address the subtle diversion of household funds to unplanned spending, which occurs each time publicly funded goods or services are accessed. 
Table 4 Multivariate ordered logistic regression analysis of food security in Sub-Sahara Africa

\begin{tabular}{|c|c|c|c|c|c|c|}
\hline & Model XVII & Model XVIII & Model XIX & Model XX & Model XXI & Model XXII \\
\hline \multirow[t]{2}{*}{ Bribery: at least once } & $1.523 * * *$ & $1.234 * * *$ & & $1.472 * * *$ & $1.270 * * *$ & \\
\hline & -0.02 & -0.039 & & -0.02 & -0.04 & \\
\hline \multirow[t]{2}{*}{ Bribe*Asset } & & $1.289 * * *$ & & & $1.195 * * *$ & \\
\hline & & -0.044 & & & -0.041 & \\
\hline \multicolumn{7}{|l|}{ Frequency of bribery [ref: never] } \\
\hline \multirow[t]{2}{*}{ Once or twice } & & & $1.243 * * *$ & & & $1.210 * * *$ \\
\hline & & & -0.019 & & & -0.019 \\
\hline \multirow[t]{2}{*}{ A few times } & & & $1.392 * * *$ & & & $1.373 * * *$ \\
\hline & & & -0.028 & & & -0.028 \\
\hline \multirow[t]{2}{*}{ Often } & & & $1.557 * * *$ & & & $1.530 * * *$ \\
\hline & & & -0.037 & & & -0.037 \\
\hline \multirow[t]{2}{*}{ No contact in the past year } & & & 0.989 & & & 1.016 \\
\hline & & & -0.017 & & & -0.018 \\
\hline \multirow[t]{2}{*}{ Urban } & $0.767 * * *$ & $0.768 * * *$ & $0.765 * * *$ & $0.757 * * *$ & $0.756^{* * *}$ & $0.756 * * *$ \\
\hline & -0.009 & -0.01 & -0.009 & -0.009 & -0.01 & -0.01 \\
\hline \multirow[t]{2}{*}{ Household size } & $1.008 * * *$ & $1.008^{* * *}$ & $1.007 * * *$ & $0.992 * * *$ & $0.992 * * *$ & $0.992 * * *$ \\
\hline & -0.002 & -0.002 & -0.002 & -0.002 & -0.002 & -0.002 \\
\hline \multirow[t]{2}{*}{ Asset } & $0.604 * * *$ & $0.571 * * *$ & $0.604 * * *$ & $0.595 * * *$ & $0.573 * * *$ & $0.595^{* * *}$ \\
\hline & -0.009 & -0.01 & -0.009 & -0.009 & -0.01 & -0.009 \\
\hline \multirow[t]{2}{*}{ Female } & $0.945 * * *$ & $0.946 * * *$ & $0.945 * * *$ & $0.947 * * *$ & $0.948 * *$ & $0.948 * * *$ \\
\hline & -0.011 & -0.011 & -0.011 & -0.011 & -0.011 & -0.011 \\
\hline Age [ref: Over 65] & $0.918 * * *$ & $0.916 * * *$ & $0.923 * * *$ & $0.885^{* * *}$ & $0.884 * * *$ & $0.889 * * *$ \\
\hline \multirow[t]{2}{*}{$18-25$} & -0.026 & -0.026 & -0.026 & -0.025 & -0.025 & -0.025 \\
\hline & $1.119 * * *$ & $1.117 * * *$ & $1.124 * * *$ & $1.067 * *$ & $1.066^{* *}$ & $1.072 * *$ \\
\hline \multirow[t]{2}{*}{$26-35$} & -0.031 & -0.031 & -0.031 & -0.029 & -0.029 & -0.03 \\
\hline & $1.189 * * *$ & $1.189 * * *$ & $1.193 * * *$ & $1.145^{* * *}$ & $1.145^{* * *}$ & $1.148 * * *$ \\
\hline \multirow[t]{2}{*}{$36-45$} & -0.033 & -0.033 & -0.034 & -0.032 & -0.032 & -0.033 \\
\hline & $1.117 * * *$ & $1.117 * * *$ & $1.122 * * *$ & $1.099 * * *$ & $1.010 * * *$ & $1.105^{* * *}$ \\
\hline \multirow[t]{2}{*}{$46-55$} & -0.033 & -0.033 & -0.033 & -0.033 & -0.033 & -0.033 \\
\hline & $1.079 * *$ & $1.080 * * *$ & $1.081 * *$ & $1.080 * *$ & $1.081 * *$ & $1.082 * *$ \\
\hline $56-65$ & -0.034 & -0.034 & -0.034 & -0.0342 & -0.034 & -0.034 \\
\hline \multicolumn{7}{|l|}{ Employment [ref: No (not looking)] } \\
\hline \multirow[t]{2}{*}{ No (looking) } & $1.238 * * *$ & $1.238 * * *$ & $1.234 * * *$ & $1.226 * * *$ & $1.226 * * *$ & $1.224 * * *$ \\
\hline & -0.018 & -0.018 & -0.017 & -0.018 & -0.018 & -0.018 \\
\hline \multirow[t]{2}{*}{ Yes, part-time } & 0.97 & $0.969 *$ & $0.945^{*}$ & 1.004 & 1.003 & 1.001 \\
\hline & -0.019 & -0.018 & -0.018 & -0.019 & -0.019 & -0.019 \\
\hline \multirow[t]{2}{*}{ Yes, full time } & $0.629 * * *$ & $0.629 * * *$ & $0.629 * * *$ & $0.663 * * *$ & $0.662 * * *$ & $0.664 * * *$ \\
\hline & -0.01 & -0.01 & -0.01 & -0.011 & -0.011 & -0.011 \\
\hline \multicolumn{7}{|l|}{ Education [ref: No formal schooling] } \\
\hline Informal schooling only & $0.925 * * *$ & $0.925 * * *$ & $0.929 * * *$ & $0.905 * * *$ & $0.905 * * *$ & $0.910 * * *$ \\
\hline & -0.026 & -0.026 & -0.026 & -0.025 & -0.025 & -0.026 \\
\hline Some primary schooling & $0.839 * * *$ & $0.839 * * *$ & $0.840 * * *$ & $0.916 * * *$ & $0.916 * * *$ & $0.918 * * *$ \\
\hline & -0.017 & -0.017 & -0.017 & -0.018 & -0.018 & -0.018 \\
\hline Primary school completed & $0.596 * * *$ & $0.597 * * *$ & $0.596 * * *$ & $0.663 * * *$ & $0.664 * * *$ & $0.665^{* * *}$ \\
\hline & -0.013 & -0.013 & -0.013 & -0.015 & -0.015 & -0.015 \\
\hline Some secondary school/high school & $0.534 * * *$ & $0.535 * * *$ & $0.534 * * *$ & $0.582 * * *$ & $0.583 * * *$ & $0.583 * * *$ \\
\hline & -0.011 & -0.011 & -0.011 & -0.012 & -0.012 & -0.012 \\
\hline Secondary school completed/high school & $0.425 * * *$ & $0.425 * * *$ & $0.423 * * *$ & $0.457 * * *$ & $0.457 * * *$ & $0.456^{* * *}$ \\
\hline & -0.009 & -0.01 & -0.009 & -0.01 & -0.01 & -0.01 \\
\hline Post-secondary qualifications, not univ & $0.335 * * *$ & $0.335 * * *$ & $0.334 * * *$ & $0.362 * * *$ & $0.362 * * *$ & 0.363 \\
\hline
\end{tabular}


Table 4 (continued)

\begin{tabular}{|c|c|c|c|c|c|c|}
\hline & Model XVII & Model XVIII & Model XIX & Model XX & Model XXI & Model XXII \\
\hline & -0.01 & -0.01 & -0.01 & -0.011 & -0.011 & -0.011 \\
\hline \multirow[t]{2}{*}{ Some university } & $0.412 * * *$ & $0.412 * * *$ & $0.410 * * *$ & $0.422 * * *$ & $0.422 * * *$ & $0.422 * * *$ \\
\hline & -0.015 & -0.015 & -0.015 & -0.016 & -0.016 & -0.016 \\
\hline \multirow[t]{2}{*}{ University completed } & $0.295 * * *$ & $0.295^{* * *}$ & $0.293 * * *$ & $0.308 * * *$ & $0.307 * * *$ & $0.307 * * *$ \\
\hline & -0.011 & -0.011 & -0.011 & -0.012 & -0.012 & -0.012 \\
\hline \multirow[t]{2}{*}{ Post-graduate } & $0.189 * * *$ & $0.189 * * *$ & $0.188 * * *$ & $0.212 * * *$ & $0.211 * * *$ & $0.212 * * *$ \\
\hline & -0.018 & -0.018 & -0.018 & -0.02 & -0.02 & -0.02 \\
\hline \multicolumn{7}{|c|}{ Community membership [Ref: Not a member] } \\
\hline \multirow[t]{2}{*}{ Inactive member } & $1.185 * * *$ & $1.184 * * *$ & $1.181 * * *$ & $1.146^{* * * *}$ & $1.146 * * *$ & $1.144 * * *$ \\
\hline & -0.019 & -0.02 & -0.02 & -0.019 & -0.019 & -0.019 \\
\hline \multirow[t]{2}{*}{ Active member } & $1.091 * * *$ & $1.091 * * *$ & $1.084 * * *$ & $1.060 * * *$ & $1.060 * * *$ & $1.054 * * *$ \\
\hline & -0.016 & -0.016 & -0.016 & -0.016 & -0.016 & -0.015 \\
\hline \multirow[t]{2}{*}{ Official member } & $1.178^{* * *}$ & $1.178 * * *$ & $1.171^{* * *}$ & $1.120 * * *$ & $1.120 * * *$ & $1.115^{* * *}$ \\
\hline & -0.029 & -0.029 & -0.029 & -0.028 & -0.028 & -0.027 \\
\hline \multirow[t]{2}{*}{ Constant cut 1} & $0.648 * * *$ & $0.623 * * *$ & $0.644 * * *$ & $0.515^{* * *}$ & $0.504 * * *$ & $0.519 * * *$ \\
\hline & -0.039 & -0.04 & -0.041 & -0.054 & -0.054 & -0.056 \\
\hline \multirow[t]{2}{*}{ Constant cut 2} & $1.254 * * *$ & $1.205^{* * *}$ & 1.247 & 1.008 & 0.986 & 1.017 \\
\hline & -0.039 & -0.04 & -0.041 & -0.054 & -0.054 & -0.056 \\
\hline \multirow[t]{2}{*}{ Constant cut 3} & $4.103 * * *$ & $3.937 * * *$ & $4.078 * * *$ & $3.344 * * *$ & $3.268 * * *$ & $3.377 * *$ \\
\hline & -0.039 & -0.04 & -0.041 & -0.0544 & -0.055 & -0.06 \\
\hline \multirow[t]{2}{*}{ Constant cut 4} & $27 . .266^{* * *}$ & $26.112 * * *$ & $27.104 * * *$ & $22.411 * * *$ & $21.898 * * *$ & $22.670 * * *$ \\
\hline & -0.043 & -0.043 & -0.044 & -0.057 & -0.057 & -0.058 \\
\hline Year effect & Yes & Yes & Yes & Yes & Yes & Yes \\
\hline Region effect & Yes & Yes & Yes & No & No & No \\
\hline Country effect & No & No & No & Yes & Yes & Yes \\
\hline Pseudo R2 & 0.044 & 0.044 & 0.045 & 0.052 & 0.052 & 0.053 \\
\hline LR Chi2 & $13,673.55$ & $13,728.93$ & $13,842.10$ & $16,120.59$ & $16,147.40$ & $16,288.28$ \\
\hline Log-likelihood & $-147,987.02$ & $-147,959.33$ & $-147,902.74$ & $-146,763.50$ & $-146,750.09$ & $-146,679.65$ \\
\hline Observations & 118,136 & 118,136 & 118,136 & 118,136 & 118,136 & 118,136 \\
\hline
\end{tabular}

Estimates for logistic regressions are in odds ratios

$O R$ robust standard errors are in parentheses

* Significant at the 0.10 level

${ }^{* * *}$ Significant at the 0.05 level

${ }^{* * *}$ Significant at the 0.01 level

No research is without limitations, and this paper is no exception. First, the survey did not differentiate between respondents who were forced to pay bribes from respondents who voluntarily offered it. Consequently, it is not easy to pin the outcome to either of the two categories. Second, the pooled data provided an opportunity to incorporate the year-effect; nevertheless, the survey's cross-sectional attribute makes it impossible to identify a causal relationship between the outcome variable and the primary independent variable of interest or any of the confounders. Third, the survey did not capture the monetary value of bribes paid; hence, the analysis could not explore how each unit of currency allocated to the discretionary expenditure impacts the respondent's food security status. Equally, the survey has no objective measure of income hence its exclusion in the analysis. Likewise, the lack of an objective income variable made it impossible to tease out the poor - non-poor divide.

\section{Conclusion}

This paper examined three questions about the relationship between the supply-side of bribery and household food security status. First, it investigated whether any bribe payment meddles with household food security status and how increased exposure to bribery affected respondents' food insecurity. Second, it probed how different government 
institutions affected the food security standing of bribepaying respondents and whether the likelihood of household food insecurity varied as the frequencies of bribery intensified. Third, it explored how the vulnerability created by exposure to bribery stacks up across different gradients of food insecurity.

Findings revealed that respondents who were victims of bribery were statistically significantly prone to experiencing food insecurity than their non-paying counterparts. Further, it showed that whenever the public comes into contact with public institutions, their food security status suffers. Challenges faced by food-insecure respondents who engaged in frequent bribe payments do not only differ, but the setback is also steeper for respondents who reported always being food insecure vis-à-vis their food-secure counterparts. These findings point to one thing: that public institutions in SubSaharan Africa are not shaping developmental pathways. Instead, they create insurmountable challenges and a bureaucratic bottleneck for the vulnerable populations, which invariably pressurize them to choose public services over food.

Future research might employ a longitudinal survey to underscore the causal relationship between actual bribery and household food security status in Sub-Saharan Africa. Another question worth examining is whether victims of bribery that experienced one form of food insecurity would still bribe when in need of another government service.

\section{Appendix 1 Regional distribution of police roadblocksby food security in SSA}

Author's contribution Not applicable.

Funding Not applicable.

Data availability The data used for this study is available from the author upon request.

Code availability The code used to obtain the findings of this study is available from the author upon request.

\section{Declarations}

Conflict of interest The author declares no conflict of interest.

\section{References}

Afrobarometer. (2011). 34 countries, Round 5, available at http://www. afrobarometer.org

Afrobarometer. (2016). 36 countries, Round 6, available at http://www. afrobarometer.org

Afrobarometer. (2018). 34 countries, Round 7, available at http://www. afrobarometer.org

Akerele, D., Momoh, S., Aromolaran, A. B., Oguntona, C. R., \& Shittu, A. M. (2013). Food insecurity and coping strategies in South-West Nigeria. Food Security, 5(3), 407-414.

Arndt, C., Davies, R., Gabriel, S., Harris, L., Makrelov, K., Robinson, S., \& Anderson, L. (2020). Covid-19 lockdowns, income distribution, and food security: An analysis for South Africa. Global Food Security, 26,100410.

Asare-Nuamah, P. (2021). Climate variability, subsistence agriculture and household food security in rural Ghana. Heliyon, 7(4), e06928.

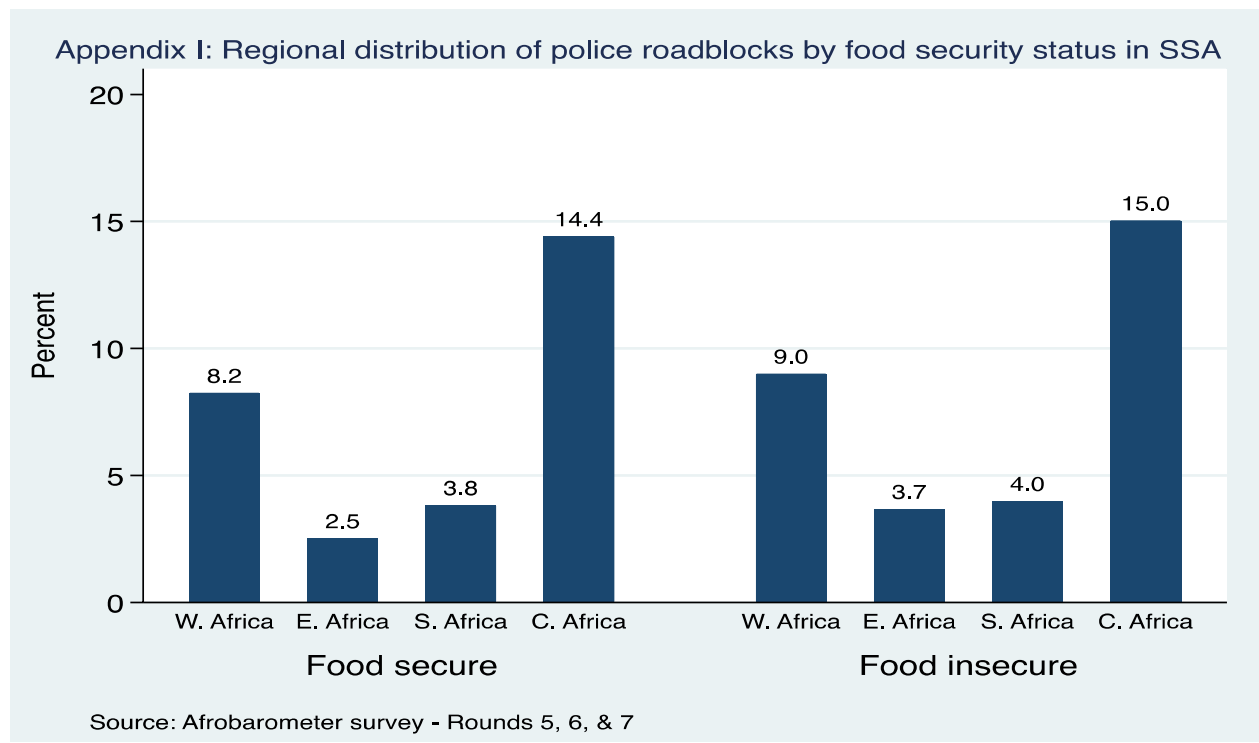


Atuoye, K. N., Antabe, R., Sano, Y., Luginaah, I., \& Bayne, J. (2019). Household income diversification and food insecurity in the upper west region of Ghana. Social Indicators Research, 144(2), 899-920.

Cameron, A. C., \& Trivedi, P. K. (2005). Microeconometrics: Methods and applications. Cambridge University Press.

Cooray, A., \& Schneider, F. (2016). Does corruption promote emigration? An empirical examination. Journal of Population Economics, 29(1), 293-310.

Dahl, M., DeLeire, T., \& Mok, S. (2014). Food insufficiency and income volatility in US households: The effects of imputed income in the survey of income and program participation. Applied Economic Perspectives and Policy, 36(3), 416-437.

de Beer, H., Harmse, J., \& Mielmann, A. (2020). Why income lacks to ensure household food security: Needs and challenges identified by consumers from a rural community, South Africa. International Journal of Consumer Studies, 44(6), 521-530.

Del Ninno, C., \& Marini, A. (2005). Household's vulnerability to shocks in Zambia. World Bank Social Protection Discussion Paper, 536.

D'Souza, A., \& Jolliffe, D. (2013). Conflict, food price shocks, and food insecurity: The experience of Afghan households. Food Policy, 42, 32-47.

Etana, D., \& Tolossa, D. (2017). Unemployment and food insecurity in urban Ethiopia. African Development Review, 29(1), 56-68.

FAO, IFAD, UNICEF, WFP and WHO. (2019). The State of Food Security and Nutrition in the World 2019. Safeguarding against economic slowdowns and downturns. Rome, FAO.

FAO, IFAD, UNICEF, WFP and WHO. (2020). The State of Food Security and Nutrition in the World 2020. Transforming food systems for affordable healthy diets. Rome, FAO. https://doi.org/10. 4060/ca9692en

Fischer, J. A. (2010). Accounting for Unobserved Country Heterogeneity in Happiness Research: Country Fixed Effects versus Region Fixed Effects. MPRA Paper No. 22272. Retrieved from https:// mpra.ub.uni-muenchen.de/22272/1/MPRA_paper_22272.pdf

Foltz, J. D., \& Opoku-Agyemang, K. A. (2015). Do higher salaries lower petty corruption? A policy experiment on West Africa's highways. Unpublished Working Paper, University of WisconsinMadison and University of California, Berkeley.

George, J., Adelaja, A., \& Weatherspoon, D. (2020). Armed Conflicts and Food Insecurity: Evidence from Boko Haram's Attacks. American Journal of Agricultural Economics, 102(1), 114-131.

Gupta, S., Davoodi, H., \& Alonso-Terme, R. (2002). Does corruption affect income inequality and poverty? Economics of Governance, 3(1), 23-45.

Gupta, S., Davoodi, H., \& Tiongoson, E. (2000). Corruption and the provision of health care and education services (IMF Working Paper WP/00/116/). Retrieved from http://www.imf.org/external/ pubs/ft/wp/2000/wp00116.pdf

Guriev, S. (2004). Red tape and corruption. Journal of Development Economics, 73(2), 489-504. https://doi.org/10.1016/j.jdeveco. 2003.06.001

Helliwell, J. F. (2003). How's life? Combining individual and national variables to explain subjective wellbeing. Economic Modelling, 20, 331-360. https://doi.org/10.1016/S0264-9993(02)00057-3

Hunt, J. (2007). How corruption hits people when they are down. Journal of Development Economics, 84, 574-589. https://doi.org/10. 1016/j.jdeveco.2007.02.003

Hunt, J., \& Laszlo, S. (2005). Bribery: Who Pays, Who Refuses, What Are the Payoffs? (No. w11635). National Bureau of Economic Research.

Hunt, J., \& Laszlo, S. (2012). Is bribery really regressive? Bribery's costs, benefits, and mechanisms. World Development, 40, 355372. https://doi.org/10.1016/j.worlddev.2011.06.001

Justesen, M., \& Bjornskov, C. (2014). Exploiting the poor: Bureaucratic corruption and poverty in Africa. World Development, 58 , 106-115. https://doi.org/10.1016/j.worlddev.2014.01.002
Krishna, A. (2007). Poverty and health: Defeating poverty by going to the roots. Development, 50(2), 63-69.

Ksenia, G. (2008). Can corruption and economic crime be controlled in developing countries and if so, is it cost-effective? Journal of Financial Crime.

Leete, L., \& Bania, N. (2010). The effect of income shocks on food insufficiency. Review of Economics of the Household, 8(4), 505-526.

Mabiso, A., Cunguara, B., \& Benfica, R. (2014). Food (In) security and its drivers: Insights from trends and opportunities in rural Mozambique. Food Security, 6(5), 649-670.

Matz, J. A., Kalkuhl, M., \& Abegaz, G. A. (2015). The short-term impact of price shocks on food security-Evidence from urban and rural Ethiopia. Food Security, 7(3), 657-679.

Mbate, M. (2018). Who bears the burden of bribery? Evidence from public service delivery in Kenya. Development Policy Review, $36, \mathrm{O} 321-\mathrm{O} 340$.

Médard, J. F. (2017). Corruption in the neo-patrimonial states of SubSaharan Africa. In Political Corruption (pp. 379-402). Routledge.

Ngcamu, B. S., \& Chari, F. (2020). Drought Influences on Food Insecurity in Africa: A Systematic Literature Review. International Journal of Environmental Research and Public Health, 17(16), 5897.

Peiffer, C., \& Rose, R. (2018). Why Are the Poor More Vulnerable to Bribery in Africa? The Institutional Effects of Services, the Journal of Development Studies, 54(1), 18-29. https://doi.org/10. 1080/00220388.2016.1257121

Seligson, M. A. (2006). The Measurement and Impact of Corruption Victimization: Survey Evidence from Latin America. World Development, 34(2), 381-404.

Shleifer, A., \& Vishny, R. (1993). Corruption. Quarterly Journal of Economics, 109(August), 599-617.

Sseguya, H., Mazur, R. E., \& Flora, C. B. (2018). Social capital dimensions in household food security interventions: Implications for rural Uganda. Agriculture and Human Values, 35(1), 117-129.

Sulemana, I., Iddrisu, A. M., \& Kyoore, J. E. (2017). A micro-level study of the relationship between experienced corruption and subjective wellbeing in Africa. The Journal of Development Studies, 53(1), 138-155.

Tavits, M. (2008). Representation, corruption, and subjective wellbeing. Comparative Political Studies, 41, 1607-1630. https://doi. org/10.1177/0010414007308537

Transparency International. (2019). Corruption perceptions Index 2018. Available at: https://www.transparency.org/news/feature/ cpi2018-subsaharan-africa-regional-analysis (accessed 30 June 2020).

Verpoorten, M., Arora, A., Stoop, N., \& Swinnen, J. (2013). Selfreported food insecurity in Africa during the food price crisis. Food Policy, 39, 51-63.

Wooldridge, J. M. (2010). Econometric analysis of cross-section and panel data (2nd ed.). MIT Press.

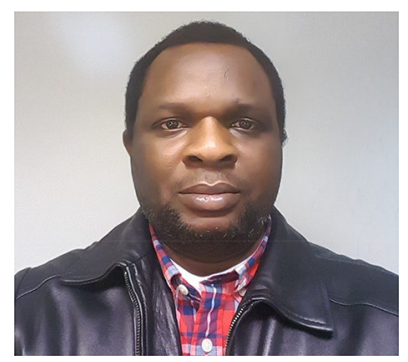

Olayemi M. Olabiyi has a $\mathrm{PhD}$ in public policy and political economy from the University of Texas at Dallas and is a faculty at SAIT School of Business. His research looks at how population health interventions interact with underlying social determinants and risk patterns to improve or worsen both overall population health and population health inequality in developed and developing regions. 